Yve Stöbel-Richter, Susanne Goldschmidt, Ada Borkenhagen, Ute Kraus \& Kerstin Weidner

\title{
Entwicklungen in der Reproduktionsmedizin - mit welchen Konsequenzen müssen wir uns auseinandersetzen?
}

Developments in reproductive medicine - what consequences do we have to deal with?

\begin{abstract}
Zusammenfassung:
Der vorliegende Artikel soll einen Überblick über verschiedene Aspekte der modernen Reproduktionsmedizin ermöglichen. Auf folgende Schwerpunkte wird deshalb genauer eingegangen: Entwicklung der medizinischen Möglichkeiten, Wissen in der Bevölkerung, Einschätzung von Chancen und Gefahren, psychologische Aspekte der Kinderlosigkeit, Belastungen während einer IVF-Behandlung, ethisch-moralische Konsequenzen der Verfahren. Darüber hinaus werden Zusammenhänge zwischen demographischer und medizinischer Entwicklung sowie die sich daraus ergebenden spezifischen psychologischen und soziologischen Perspektiven aufgezeigt. Die bisherigen Forschungsergebnisse zeigen nicht nur ein Mangel an Wissen bzgl. fortpflanzungsmedizinischer Aspekte, sondern auch übertrieben große, zum Teil unberechtigte, Hoffnungen hinsichtlich der Wirksamkeit der reproduktionsmedizinischen Verfahren. Oftmals werden hochaufwändige und kostenintensive Verfahren eingesetzt, um den (langen) Wunsch nach einem Kind zu erfüllen, allerdings ohne psychischen und sozialen Wirkfaktoren Rechnung zu tragen. Somit steht die Devise „ein Kind um jeden Preis“" seitens vieler Paare und aber auch Reproduktionsmediziner im krassen Gegensatz zu mangelnder Beratung vor, während und nach einer $\mathrm{Be}$ handlung.
\end{abstract}

\begin{abstract}
:
This report provides an overview about certain aspects of modern reproductive medicine. The following issues are prioritised: development of medical facilities, people's knowledge on reproductive medicine, appraisal of chances and risks, psychological aspects of involuntary childlessness, stresses and strains during IVFtreatment, ethical consequences of these medical procedures. Furthermore interactions between demographic and medical developments are analysed as well as resulting psychological and sociological perspectives. Previous research results present both a lack of knowledge towards reproductive medical treatments and disproportionate expectations and hope toward treatment outcome. Very often expensive and complex methods are practiced to fulfil the child wish, but mental and social aspects remain unconsidered. So the motto "a child at any cost" on the part of many involuntary childless couples as well as of some fertility doctors poses a glaring contradiction to insufficient counselling in practice prior to, during and after the treatment.
\end{abstract}


Stichworte: Reproduktionsmedizin, ungewollte Kinderlosigkeit, Fertilitätsentwicklung, Kinderwunschberatung
Key words: Reproductive medicine, involuntary childlessness, fertility development, counselling and psychological assessment in fertility medicine

\section{Fertilitätsentwicklung als demographischer Prozess}

Der gesellschaftliche Wandel in den letzten Jahrzehnten kann hinsichtlich familiensoziologischer Aspekte auch als Spannungsfeld zwischen Freiheit und Risiko umschrieben werden. Familiengründung ist nur noch eine Wahloption unter vielen, was auch die Option offen lässt, gar keine Familie zu gründen. Vielfach wird in diesem Kontext von „Bastelbiographien“ und hinsichtlich der verschiedenen Familienformen von „Patchworkfamilien“ oder auch „Wahlverwandtschaften“ gesprochen. War Elternschaft früher selbstverständlich, so wird heute mehr und mehr ein Problem daraus. Dabei sind Zögern, Abwägen und Aufschub kein privater Konflikt, sondern vielmehr Ausdruck des derzeitigen epochalen gesellschaftlichen Wandels. Dieser Wandel hat dazu geführt, dass alte Bindungen aufgelöst wurden und neue Formen des Lebenslaufs und - damit einhergehend - neue Erwartungen und Anforderungen, neue Freiräume und aber auch Abhängigkeiten entstehen. „Der Anspruch auf ein Stück eigenes Leben, der im Gefolge des Wandels entsteht [...] ist wie ein Spiegel, in dem wir den Aufbruch und Umbruch der Lebensformen der Moderne erkennen, ihre Verheißungen und Sehnsüchte, ihre Enttäuschungen und Ängste [...] und ihre Konflikte und Widersprüche, die sich hinein schieben in das Leben der Frau und in das Verhältnis zwischen Mutter und Kind“" (Beck-Gernsheim, 2006, 22 23).

Die Kultur und Strukturen einer Gesellschaft prägen somit auch die Eltern-KindBeziehungen und haben Einfluss auf die Entwicklung der Fertilität. Im sogenannten Human Development Index der Vereinten Nationen werden anhand von Pro-KopfEinkommen, Lebenserwartung und Schulbildung der Bevölkerung eines Landes Rückschlüsse auf die Pro-Kopf-Geburtenzahl gezogen. Diese sogenannte total fertility rate hängt eng mit der ökonomischen Entwicklung eines Landes zusammen. Je höher ein Land entwickelt und je modernisierter es ist, desto höher sind vielfach auch die Kosten, die durch ein Kind entstehen. Diese direkten Opportunitätskosten umfassen jene Kosten, welche für die Erziehung und Betreuung eines Kindes aufgebracht werden müssen. Darüber hinaus sind hier aber auch jene indirekten Kosten gemeint, welche daraus resultieren, dass eine Frau vor der Geburt eines Kindes berufstätig und somit ökonomisch unabhängig war und die nun aufgrund des Verdienstausfalls entstehen. Je nach den geltenden kulturellen und gesellschaftlichen Maßstäben können die Opportunitätskosten sehr hoch sein und in der Folge ein niedriges Geburtenniveau nach sich ziehen (Dickmann, 2003; BMFSFJ, 2005).

Dieser Zusammenhang ist auch erkennbar, wenn man die Fertilitätsraten, d.h. die Anzahl der Kinder je Frau im gebärfähigen Alter, im internationalen Vergleich betrachtet: Deutschland befindet sich dabei mit durchschnittlich 1,4 Kindern je Frau eher im unteren Drittel der Industrieländer. Bei der Betrachtung der Entwicklung 
der Bruttogeburtenziffer je 1000 Einwohner im internationalen Vergleich wird deutlich, wie stark die Geburten in den letzten Jahren in fast allen Industrieländern zurückgegangen sind (Tabelle 1). Wurden im Jahr 1950 in Europa noch zwischen 16 und 20 Kinder je 1000 Einwohner geboren, so sank die Zahl bis zum Jahr 2005 teilweise um mehr als die Hälfte (vgl. Hill \& Kopp, 2000; eurostat 2003, Lanzieri \& Corsini, 2006). Vor allem in den ehemals sozialistischen Staaten waren die Prozesse gravierend, beispielhaft hierfür kann die Entwicklung der Geburtenzahlen in den neuen Bundesländern zwischen 1990 und 1995 betrachtet werden.

Tabelle 1: Entwicklung der Geburtenzahlen im internationalen Vergleich (pro 1000 Einwohner)

\begin{tabular}{lcccccrrc}
\hline Land & 1950 & 1960 & 1970 & 1980 & 1990 & 1995 & 2002 & 2005 \\
\hline USA & 23,9 & 23,7 & 18,3 & 15,9 & 16,7 & 15,3 & 14,2 & k.D. \\
\hline BRD/ alte BL & 16,2 & 17,4 & 13,4 & 10,1 & 11,5 & 10,3 & G: 8,8 & G: 8,4 \\
DDR/ neue BL & 16,5 & 17,0 & 13,9 & 14,6 & 11,1 & 5,4 & & \\
Frankreich & 19,8 & 18,1 & 16,8 & 14,9 & 13,3 & 12,5 & 12,8 & 12,6 \\
Großbritannien & 15,9 & 18,2 & 16,3 & 13,4 & 13,8 & 12,6 & 11,3 & 11,9 \\
Schweden & 16,6 & 14,0 & 13,7 & 11,7 & 14,5 & 11,7 & 10,7 & 10,4 \\
Italien & 18,6 & 18,3 & 16,8 & 11,4 & 9,8 & 9,2 & 9,4 & 9,9 \\
Spanien & 20,1 & 21,5 & 19,5 & 15,3 & 10,2 & 9,1 & 10,1 & 10,9 \\
\hline Indien & 44,1 & 42,0 & 38,2 & 34,7 & 29,9 & 28,6 & 23,8 & k.D. \\
China & 43,6 & 37,8 & 30,6 & 21,0 & 21,2 & 17,8 & 13,1 & k.D. \\
\hline
\end{tabular}

k.D. = keine Daten verfügbar

Quelle: Hill \& Kopp, 2000; eurostat 2003; Lanzieri \& Corsini, 2006

Im Jahr der deutschen Vereinigung (1990) betrug die zusammengefasste Geburtenziffer für die DDR erstmals seit 1950 nur mehr knapp 1,5. Im nachfolgenden Jahr (1991) sank diese Ziffer dann weiter auf 0,98 ab. Die für 1993 berechnete zusammengefasste Geburtenziffer betrug 1,4 für Westdeutschland und nur 0,8 für Ostdeutschland. Dieser Trend setzte sich bis 1995 fort, erst seit diesem Zeitraum ist wieder ein Anstieg der Geburten zu verzeichnen, ohne dass allerdings das westdeutsche Niveau erreicht wurde. Derzeit weist Deutschland eine der weltweit niedrigsten Geburtenraten auf.

In Deutschland sind hinsichtlich der Geburtenentwicklung die folgenden Trends charakteristisch: Einerseits erfolgt ein freiwilliger Verzicht auf Kinder oder die Realisierung des Kinderwunsches wird zugunsten anderer Lebensalternativen zurückgestellt. Andererseits wird aufgrund gestiegener Opportunitätskosten sowie eines enorm hohen Erziehungsanspruchs die Kinderzahl auf eine Größe beschränkt, die den gegebenen Lebensbedingungen entspricht. Besonders Frauen mit hohem Bildungsabschluss entscheiden sich immer häufiger gegen oder sehr spät für Kinder. Auch wenn die Zahlen von 41 Prozent kinderlosen Akademikerinnen des Jahrgangs 1965 (Dickmann, 2003; Scharein \& Unger, 2005) umstritten sind (Sobotka, 2005; Neyer, 2005), so zeigt sich doch eine deutliche Entwicklung zur Kinderlosigkeit, nicht nur bei Frauen, sondern auch bei Männern und nicht nur im akademischen Bereich (DIW, 2004). Scharein und Unger (2005) legen dar, dass die Angaben zu den kinderlosen Akademikerinnen stark entsprechend der zugrunde gelegten Datenbasis (Mikrozensus oder Sozio-ökonomisches Panel - SOEP) differieren und zusätzlich da- 
von abhängen, wie der Begriff „,akademisch“ definiert wird. „Während der Mikrozensus auf sehr hohen Fallzahlen beruht (es handelt sich um eine 1\%-Stichprobe bundesdeutscher Haushalte), hat er deutliche Schwächen bei biografischen Ereignissen wie Geburten. Das Sozio-ökonomische Panel hat zwar eine deutlich kleinere Fallzahl, kann jedoch Geburten im Lebenslauf zu den befragten Frauen zuordnen und dadurch das ,tatsächliche• Geburtenverhalten abbilden“ (Scharein und Unger, 2005; 6). Berücksichtigt man hingegen die Heterogenität der Gruppe der Akademikerinnen und betrachtet darüber hinaus die gleichen Altersjahre, so differieren die Angaben kaum noch.

Eine beschränkte Kinderzahl ergibt sich auch aus der Entwicklung, dass die Entscheidung zur Elternschaft grundsätzlich nach hinten verschoben wird; das durchschnittliche Erstgraviditätsalter liegt inzwischen bei 28 Jahren. Der Anteil der sog. Spätgebärenden (Frauen über 34 Jahre) ist in den letzten zwei Jahrzehnten von 1,3\% auf $22 \%$ erheblich gestiegen. Dies führt dazu, dass inzwischen $70 \%$ aller Schwangerschaften von den Medizinern als Risikoschwangerschaften eingestuft werden, was wiederum enorme Mehrkosten und diagnostischen Aufwand nach sich zieht (Cornelißen, 2004; Saleth, 2005; Wirth \& Dümmler, 2005).

Somit ist zwar die Entscheidung für ein erstes oder zweites Kind verschiebbar, damit sinkt allerdings die Wahrscheinlichkeit, dass noch eine Entscheidung für ein drittes oder gar viertes Kind positiv ausfällt (Sobotka, 2005). In Deutschland ist die Zahl der gewünschten Kinder im Vergleich mit anderen ausgewählten europäischen Ländern am geringsten: Frauen geben eine durchschnittliche ideale Kinderzahl von 1,75, Männer von 1,59 an. In den anderen Ländern geben die Frauen Werte zwischen 1,84 (Österreich) und 2,33 (Polen), die Männer Werte zwischen 1,78 (Österreich) und 2,29 (Polen) an (Höhn, Ette \& Ruckdeschel, 2006).

Neben dem sehr hohen Anteil an Kinderlosen ist auch die Entwicklung der Familienstrukturen dadurch gekennzeichnet, dass jene Frauen, die sich für eine Familie entscheiden, vielfach mehr als ein Kind bekommen und somit die durchschnittliche Geburtenrate teilweise ausgleichen. Somit findet eine Polarisierung der Familienstrukturen statt: Einerseits ist ein deutlicher Rückgang der Ein-Kind-Familie sowie eine Zunahme der Kinderlosigkeit zu verzeichnen, andererseits entscheiden sich nach wie vor 8 Prozent für vier und mehr Kinder (Dickmann, 2003, vgl. Abbildung 1). Diese Prozesse werden in der Soziologie auch als bimodale Verteilung der Kinderzahlen bezeichnet: entweder es erfolgt kein Übergang zur Elternschaft (keine Kinder) oder aber, wenn denn einmal die Entscheidung zur Elternschaft getroffen wurde, erfolgt dies sehr häufig für zwei Kinder. Die Elternschaft mit nur einem Kind ist hingegen seltener. Hierbei muss allerdings nach Bildungsgrad und Geburtsjahrgang sowie nach Region (Ost/ West) unterschieden werden (Grünheid, 2004; Huinink, 2002). 
$A b b$ 1: Entwicklung der Familienstrukturen in Deutschland - Anteile der Frauen nach Anzahl der Kinder in Prozent

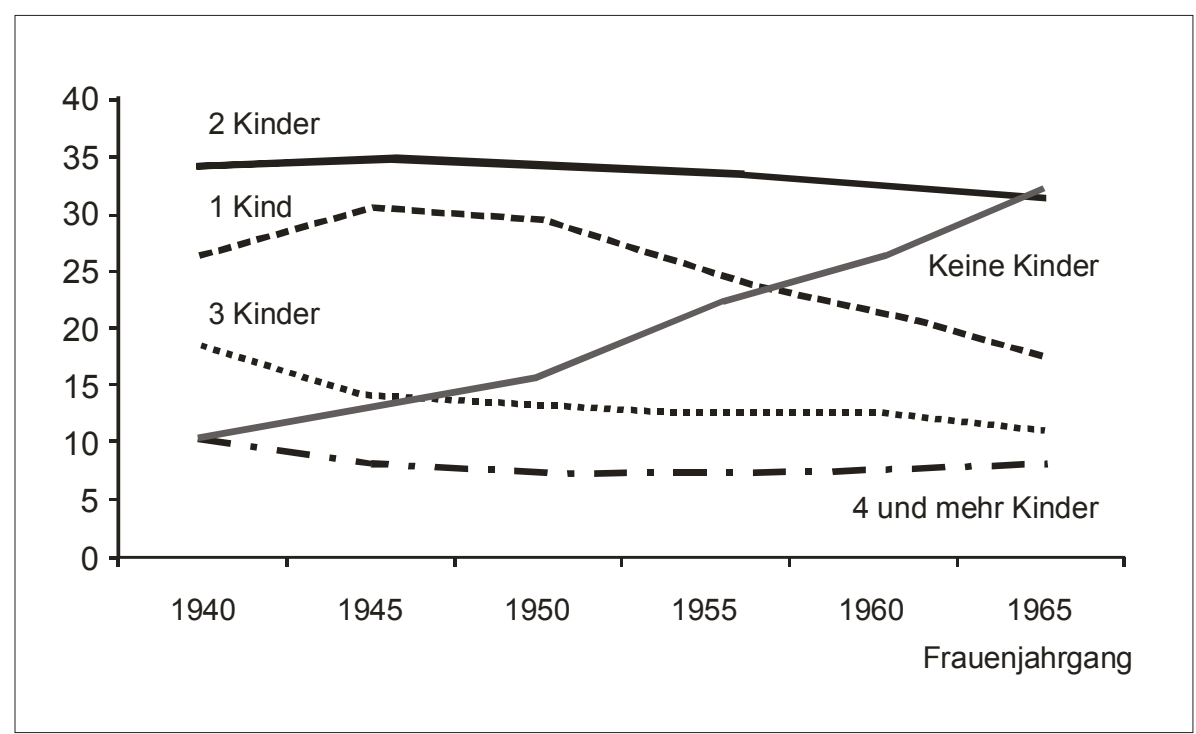

Quelle: Dickmann, 2003, 20

\section{Gewollte und ungewollte Kinderlosigkeit}

Grundsätzlich kann in den letzten Jahren ein Anstieg der Zahl der Kinderlosen registriert werden. Schätzungen für den Geburtsjahrgang 1970 gehen von ca. 30\% kinderlosen Frauen für diesen Jahrgang aus (Dickmann, 2003). Allerdings können genaue Zahlen zur Kinderlosigkeit erst nach Abschluss der fertilen Phase, also für Frauen, die 45 Jahre und älter sind, ermittelt werden. In der jüngsten Veröffentlichung des Bundesinstitutes für Bevölkerungsforschung werden für den Jahrgang 1955, also für jene Frauen, die ihre fertile Phase definitiv abgeschlossen haben, 22\% als kinderlos angegeben, in anderen ausgewählten europäischen Ländern lag die Rate zwischen 8\% (Frankreich) und 17\% (Niederlande und Großbritannien) (Höhn, Ette \& Ruckdeschel, 2006).

Bei der Angaben dieser Zahlen erfolgt jedoch in der Regel keine Unterscheidung zwischen gewollter und ungewollter Kinderlosigkeit. Vor allem die für den Anteil der ungewollt Kinderlosen angegebenen Zahlen sind mit Raten von 10-15 Prozent oftmals zu hoch (Crosignani \& Rubin, 1996; Brähler \& Stöbel-Richter, 2002). Genauere Schätzungen gehen davon aus, dass:

- $20-30 \%$ aller Paare einmal in ihrem Leben unter verminderter Fruchtbarkeit leiden, d.h. innerhalb eines Jahres bei ungeschütztem Koitus nicht schwanger werden (Küppers-Chinnow \& Karmaus, 1997; Ittner, Himmel \& Kochen, 2000; ESHRE Capri Workshop, 2001), 
- 6-9\% aller Paare in Mitteleuropa ungewollt kinderlos sind und eine Behandlung wünschen,

- sowie ca. 3\% dauerhaft ungewollt kinderlos bleiben (Wischmann et al., 2004; Stöbel-Richter \& Brähler, 2005).

Die Entscheidung für oder gegen Kinder ist ein Prozess, der verschiedene Stadien durchlaufen kann; dabei liegt häufig zunächst eine gewollte Kinderlosigkeit vor, die später in eine ungewollte übergehen kann (vgl. Dorbritz, 2005; Kemkes-Grottenthaler, 2003). Nach dem aktuellen Kinderwunsch befragt, gaben in der unten ausführlicher beschriebenen Repräsentativbefragung aus dem Jahr 2003 von 2110 Männern und Frauen zwischen 18 und 50 Jahren lediglich 3,2\% aller ProbandInnen einen starken bzw. sehr starken aktuellen Kinderwunsch an. 33\% derjenigen, die generell noch ein Kind wollen bzw. noch ambivalent sind, gaben an, sich derzeit kein Kind zu wünschen (Stöbel-Richter \& Brähler, 2006).

Grundsätzlich sollte bei der Frage nach dem aktuellen Kinderwunsch zwischen primärem und weiterem Kinderwunsch unterschieden werden, da es sich bei kinderlosen Personen immer um einen antizipierten Kinderwunsch handelt, während der Wunsch nach einem weiteren Kind auf eigenen Erfahrungen gründet. So ist der Kinderwunsch als solcher, vor allem vor der Erstelternschaft, häufig sehr ambivalent besetzt - vieles spricht dafür, sich ein Kind zu wünschen, gleichzeitig existieren Ängste und Befürchtungen, die vor allem in der psychologischen Literatur sehr ausführlich aufgearbeitet sind (vgl. Gloger-Tippelt, Gomille \& Grimmig, 1993; 1994, aber auch Stöbel-Richter, 2000). Dementsprechend häufig wird der Kinderwunsch in höhere Lebensalter verschoben; die Frauen sind heute bei der Geburt ihres ersten Kindes im Durchschnitt 28 Jahre alt, Tendenz steigend.

Generell ist der Wunsch nach einem Kind eng mit dem Prozess des ElternWerdens verbunden; je nach Standpunkt des Betrachters kann dieser Prozess mehr oder weniger individuumsbezogen interpretiert werden, womit die Thematik des Kinderwunsches sowohl Fragen der Realisierung durch Geburten als auch Fragen der Negierung durch Schwangerschaftsabbrüche (Henning \& Henning, 1992) umfasst. Der individuelle Wunsch nach einem Kind ist ein historisch relativ neues Phänomen, da erst die Verfügbarkeit zuverlässiger Verhütungsmethoden und die damit verbundene Trennung von Sexualität und Fortpflanzung eine reale Entscheidungsfreiheit für bzw. gegen ein (weiteres) Kind ermöglichten (Frick-Bruder \& Schütt, 1992). Noch zu Beginn des 20. Jahrhunderts war es selbstverständlich, Kinder zu bekommen, die Frage: Ein Kind - ja oder nein? stellte sich als solche nicht. Empfängnis, Geburt und Tod waren in eine religiöse und soziale Ordnung eingebunden (Gloger-Tippelt, Gomille \& Grimmig, 1993). Obwohl es bereits im Zuge der Industrialisierung zu grundlegenden Veränderungen der Familienformen kam, war die Entscheidungsfrage für oder gegen Kinder als solche irrelevant.

Im individuellen Kinderwunsch spiegeln sich bei Männern und Frauen vielfältige bewusste und unbewusste Wünsche wider (vgl. Beutel, 2002; Beck \& BeckGernsheim, 1990; Brähler, 1990; Burkart, 1994; Gloger-Tippelt et al., 1994; Kühler, 1989). Grundsätzlich ist die Bedeutung des Kinderwunsches für beide Geschlechter sehr vielschichtig: „Die Psychoanalytische Theorie beschreibt grundlegende Reifungsprozesse, die in den Wunsch nach Elternschaft münden. Empirische Untersu- 
chungen weisen aus, dass der Wunsch nach einem Kind geprägt ist von gesellschaftlichen Erwartungen und Normen. Darüber hinaus kann der Kinderwunsch einen Lösungsversuch für intrapsychische oder psychosoziale Konflikte darstellen“ (Brähler, 1990, 35).

In neueren psychologischen Ansätzen wird der Kinderwunsch als Zusammenfassung verschiedener mehr oder weniger bewusster Motive zu Schwangerschaft, Geburt und Elternschaft gekennzeichnet, welche durch biologische und intrapsychische Voraussetzungen der Person sowie durch Sozialisation und gesellschaftliche Normen determiniert werden (Mittag \& Jagenow, 1985; Gloger-Tippelt et al., 1993; Beutel, 2002; Stammer et al., 2002). Vielfach wird davon ausgegangen, dass diese Aspekte sowohl bejahenden als auch verneinenden Charakter haben und der Kinderwunsch damit ambivalent ausgeprägt ist.

Ruckdeschel $(2005,4)$ schreibt zum Kinderwunsch: „Der Kinderwunsch ist ein seit langem bekanntes und umstrittenes Maß zur Prognose von Geburtenzahlen. Als sicher kann gelten, dass er Informationen über die grundlegende Bereitschaft, Kinder zu bekommen, liefert. Diese Bereitschaft ist in den letzten 15 Jahren zurückgegangen, wobei vor allem die gewünschte Kinderlosigkeit zugenommen hat. Insgesamt lässt sich ein Polarisierungsphänomen beobachten, das dem tatsächlichen Geburtenverhalten gleicht und für die alten Bundesländer stärker ausgeprägt ist als für die neuen.“

Viele Untersuchungen zeigen, dass die intendierte Kinderzahl im Lebenslauf nicht konstant ist. So zeigten die Ergebnisse der Bamberger Panel-Studie (Schneewind et al. 1992, 346; vgl. auch Schneewind et al., 1997), dass auffällig viele Ehepaare innerhalb einer relativ kurzen Phase der Ehe ihre Kinderwünsche verändert hatten - in Form von einem Aufschub, durch eine zeitliche Konkretisierung, die zeitliche Vorverlagerung der Realisierung der Elternschaft oder die Veränderung der gewünschten Kinderzahl. „Die Ausprägung des Kinderwunsches ist in den meisten Fällen kein Ergebnis einer endgültig feststehenden und punktuellen Entscheidung, sondern eine veränderliche Option" (Schneewind et al. 1992, 346). Turchi (1991) beschreibt, dass Änderungen der persönlichen Lebensumstände auch zu einer Veränderung der Familienplanung führen können und dass sich auch die Geburt des ersten Kindes auf den Wunsch nach weiteren Kindern auswirken kann.

Die Realisierung des Kinderwunsches hängt aber auch mit der vermeintlichen Planbarkeit von Schwangerschaften zusammen, von welcher, vor allem beim ersten Kind a priori ausgegangen wird. In all unseren Untersuchungen zeigte sich jedoch immer wieder, dass ein großer Anteil an Schwangerschaften ungeplant zustande kommt (Brähler \& Stöbel-Richter, 2003; Stöbel-Richter, 2007): So gaben 1999 in einer repräsentativen Erhebung zu Elternschaft und Kinderwunsch von denjenigen Probanden, welche in den letzten zwei Jahren ein Kind bekommen hatten, rund ein Drittel in den alten und knapp die Hälfte der Probanden in den neuen Bundesländern an, dass die Schwangerschaft ungeplant zustande gekommen sei.

Von 518 Frauen zwischen 18 und 45 Jahren, die in einer Paarbefragung zusammen mit ihren Partnern im Jahr 2003 befragt wurden, gaben 24\% an, in den letzten zwei Jahren schwanger gewesen zu sein. Davon war bei $72 \%$ die Schwangerschaft geplant, bei 28\% ungeplant zustande gekommen (Stöbel-Richter, 2006).

In der Sächsischen Längsschnittstudie, welche ca. 500 Personen seit 20 Jahren jährlich befragt, sind die Probanden inzwischen (2007) 34 Jahre alt und damit mit- 
ten im Prozess der Familiengründung. Betrachtet man dabei die Planung von Schwangerschaften in den letzten zwei Jahren, so zeigen die Ergebnisse einerseits, dass Schwangerschaften zwar geplant werden, aber nicht zustande kommen (bei der Hälfte derjenigen TeilnehmerInnen, die im Jahr 2004 eine Schwangerschaft geplant hatten) und dass andererseits bei einem Drittel (im Jahr 2006) die Schwangerschaft ungeplant zustande kam. Beide Ergebnisse sprechen dafür, dass der Übergang zur Elternschaft nicht einer vollständig intendierten Planung unterliegt, sondern eher ein Prozess des Abwägens stattfindet, dem einerseits Unsicherheiten, Ambivalenzen und Ängste und andererseits emotionale Wünsche zugrunde liegen (Stöbel-Richter et al., 2006a; Stöbel-Richter, 2007).

\section{Entwicklung der medizinischen Möglichkeiten}

Durch die schrittweise Entkopplung von Sexualität, Befruchtung und Fortpflanzung kommt es zu nachhaltigen Veränderungen, welche das Leben zukünftiger Generationen entscheidend bestimmen werden.

Die wenigsten wissen, dass bereits im Jahr 1878 (!) erste Experimente durchgeführt wurden, die der späteren Entwicklung der In-vitro-Fertilisation (IVF) dienten. Ein weiterer Meilenstein war die Entdeckung der Spermienflüssigkeit 1951, sowie zahlreiche Tierversuche mit Hamstern, Mäusen und Kaninchen... Die 1950er und 1960er Jahre waren das „Goldene Zeitalter“ der IVF (Bavister, 2002), erste Erfolge zeichneten sich bei verschiedenen Spezies ab; erstmalig konnten Eizellen von verschiedenen Säugetieren außerhalb des Körpers, in vitro, befruchtet werden. Diese entwickelten sich nach dem „Einpflanzen“ normal und lebende Tiere wurden geboren. Die erste erfolgreiche Befruchtung menschlicher Eizellen wurde bereits 1969 verzeichnet (Edwards et al., 1969; Bavister, 2002), dennoch dauerte es noch einmal neun Jahre, bis das erste Kind nach einer In-vitro-Fertilisation geboren wurde.

Der erfolgreiche Einsatz der IVF im Jahre 1978 ermöglichte eine Abkopplung der Fortpflanzung von der Sexualität, denn das (zukünftige) Kind wurde im Reagenzglas, durch Dritte (Biologen, Fortpflanzungsmediziner) gezeugt. Mit der Entwicklung von ICSI (Intracytoplasmatische Spermieninjektion) im Jahre 1991 wurde die Fortpflanzung auch ohne die Fruchtbarkeit des Mannes möglich, da bei diesem Verfahren Spermien des Mannes direkt aus dem Hodensack punktiert und im Reagenzglas mit der Eizelle der Frau zusammen gebracht werden. Durch die neuesten Entwicklungen wie die PID (Präimplantationsdiagnostik) und die damit mögliche Rohstoffgewinnung aus embryonalen Stammzellen wird inzwischen die Befruchtung auch ohne Fortpflanzung angedacht. Welche Folgen sich aus therapeutischem Klonen oder Klonen zum Zwecke der Reduplikation von Personen ergeben, ist heute überhaupt noch nicht abschätzbar.

In Deutschland sind die Bestimmungen durch das Embryonenschutzgesetz streng geregelt. Aber mit seinen Verboten für z.B. Eizellspende, Leihmutterschaft, Stammzellforschung und Präimplantationsdiagnostik steht Deutschland vielfach allein. Vor allem in den USA ist die Anwendung der o.g. Verfahren erlaubt und somit möglich. Aber auch in Deutschland geraten Politiker zunehmend unter Druck, ob die strengen 
Auflagen des Embryonenschutzgesetzes gelockert werden sollen, nicht zuletzt, um im internationalen Wettbewerb mithalten zu können. Vor dem Hintergrund der nach wie vor aktuellen Debatte um die Genehmigung der Präimplantationsdiagnostik (PID) ist an dieser Stelle daran zu erinnern, dass bei der Einführung der IVF die Indikation für die Anwendung dieser Technik zunächst auch relativ eng gefasst war; angedacht war der Einsatz des Verfahren bei Frauen mit verschlossenen Eileitern. Inzwischen wird in vielen Zentren jede Frau behandelt, wenn sie ihren Wunsch nur explizit zum Ausdruck bringt und es bezahlen kann. Vielerorts gelten reproduktionsmedizinische Verfahren wie IVF und ICSI, aber auch die Anwendung pränataldiagnostischer Verfahren als selbstverständliche Dienstleistung (Sorg \& Fränznick, 2002). Seitens vieler Ärzte wurde dabei lange Zeit lediglich die somatische Ebene beachtet. Inzwischen setzt sich dank einer psychosomatisch orientierten Gynäkologie mehr und mehr durch, auch psychische, berufliche und partnerschaftliche Aspekte im Kontext mit ungewollter Kinderlosigkeit zu berücksichtigen (vgl. hierzu die Ausführungen von Strauß, Brähler \& Kentenich, 2004; Sakolos et al., 2004; Wischmann, 2003; Malin et al., 2001; Felder et al., 2002; Siedentopf et al. 2001).

\section{Wahrnehmung von Entwicklungen der modernen Reproduktionsmedizin in der Bevölkerung}

Die im Folgenden vorgestellten Ergebnisse stammen aus der vom BMBF geförderten Verbundstudie „Einstellungen und Wissen zu kontroversen medizinischen Fragen der Reproduktionsmedizin und Präimplantationsdiagnostik“ (BMBF-Förderkennzeichen 01GP0205/0255). Im Leipziger Teilprojekt war die Erhebung von Einstellungen $\mathrm{zu}$ modernen reproduktionsmedizinischen Verfahren in der deutschen Allgemeinbevölkerung, im Berliner Teilprojekt die Erhebung von Einstellungen zu modernen reproduktionsmedizinischen Verfahren bei Paaren, die eine Kinderwunschbehandlung begonnen hatten, Inhalt der Studie. Im Auftrag der Universität Leipzig wurden durch das Markt- und Meinungsforschungsinstitut USUMA (Berlin) im November 2003 im Rahmen einer Mehrthemenumfrage 416 Ostdeutsche und 1694 Westdeutsche im Alter von 18 bis 50 Jahren befragt. Das durchschnittliche Alter der Befragten betrug 35,8 Jahre, die Standardabweichung 9,1 Jahre. Die Stichprobe bestand aus 929 Männern und 1181 Frauen, eine genauere Stichprobenbeschreibung kann der folgenden Tabelle 2 entnommen werden. Die Personen wurden von geschulten Interviewern zu Hause aufgesucht und dort befragt (Face-toface-Interviews). Die Zufallsauswahl der Haushalte erfolgte nach einem RandomRoute-Verfahren, wobei die im Haushalt zu befragende Zielperson ebenfalls nach dem Zufallsprinzip ermittelt wurde. Die Repräsentativität der Stichprobe wurde durch die Ziehung von ADM-(Arbeitskreis Deutscher Marktforscher)-Stichproben gesichert (vgl. ADM, 1979). Um eine Repräsentativität der Stichprobe bezüglich Alter und Geschlecht zu gewährleisten, wurden die vorhandenen Werte so gewichtet, dass sie in diesen Merkmalen der Grundgesamtheit entsprechen (USUMA, persönliche Mitteilung). 
Tabelle 2: Stichprobencharakteristik

\begin{tabular}{|c|c|c|c|c|}
\hline & & Gesamt & Männer & Frauen \\
\hline \multirow[t]{2}{*}{ Alter (in Jahren) } & MW & 35.8 & 35.4 & 36.0 \\
\hline & SD & 9.1 & 9.3 & 8.8 \\
\hline \multirow{5}{*}{ Familienstand } & Verheiratet, zusammenlebend & $1138(53.9 \%)$ & $458(49.3 \%)$ & $680(57.6 \%)$ \\
\hline & Verheiratet, getrennt lebend & $29 \quad(1.4 \%)$ & $13(1.4 \%)$ & $16 \quad(1.4 \%)$ \\
\hline & ledig & $712(33.7 \%)$ & $384(41.3 \%)$ & $328(27.8 \%)$ \\
\hline & geschieden & $194 \quad(9.2 \%)$ & $67 \quad(7.2 \%)$ & $127(10.7 \%)$ \\
\hline & verwitwet & $37 \quad(1.8 \%)$ & $7 \quad(0.8 \%)$ & $30 \quad(2.5 \%)$ \\
\hline \multirow[t]{2}{*}{ Kinder } & ja & $1275(60.4 \%)$ & $472(50.8 \%)$ & $803(68.0 \%)$ \\
\hline & nein & $835(39.6 \%)$ & $457(49.2 \%)$ & $378 \quad(32.0 \%)$ \\
\hline \multirow{7}{*}{$\begin{array}{l}\text { Höchster } \\
\text { Abschluss }\end{array}$} & Ohne Abschluss & $19 \quad(0.9 \%)$ & $5 \quad(0.5 \%)$ & $14 \quad(1.2 . \%)$ \\
\hline & Haupt-/Volksschulabschluss & $625(29.6 \%)$ & $293(31.5 \%)$ & $332(28.1 \%)$ \\
\hline & $\begin{array}{l}\text { Mittlere Reife/ } \\
\text { Realschulabschluss }\end{array}$ & $974(46.2 \%)$ & $385(41.5 \%)$ & 589 (49.9\%) \\
\hline & $\begin{array}{l}\text { FS-Abschluss (o. Anerkennung } \\
\text { als FH-Abschluss) }\end{array}$ & $90 \quad(4.3 \%)$ & $34 \quad(3.7 \%)$ & $56 \quad(4.7 \%)$ \\
\hline & $\begin{array}{l}\text { Hochschulreife (Abitur o. abge- } \\
\text { schlossenes Studium) }\end{array}$ & $231(11.0 \%)$ & $101(10.9 \%)$ & $130(11.0 \%)$ \\
\hline & $\begin{array}{l}\text { Uni-/Hoch-/ Fachhoch- } \\
\text { schulabschluss }\end{array}$ & $151 \quad(7.2 \%)$ & $97(10.4 \%)$ & $54 \quad(4.6 \%)$ \\
\hline & Schüler/Student & $20 \quad(1.0 \%)$ & $14 \quad(1.5 \%)$ & $6 \quad(0.5 \%)$ \\
\hline
\end{tabular}

Vielfach gehen Paare davon aus, dass ihnen mit den Möglichkeiten der modernen Reproduktionsmedizin eine zuverlässige und zeitlich fast unbegrenzte Hilfe bei der Einlösung ihres unerfüllten Kinderwunsches zur Verfügung steht (Onnen-Isemann, 2000; Brähler \& Stöbel-Richter, 2002). Diese Annahme basiert allerdings häufig auf mangelhaftem Wissen über die Zusammenhänge zwischen Alter und Fruchtbarkeit. In der o.g. Repräsentativerhebung aus dem Jahr 2003 wurde gefragt, ab welchem Alter die Fruchtbarkeit der Frau langsam abnimmt. Dass dies bereits mit 25 Jahren der Fall ist, wussten die wenigsten der Befragten. Knapp ein Drittel der Frauen und Männer gaben ab 40 Jahre an, etwas weniger als ein Drittel sogar noch später (vgl. Abbildung 2).

Grundsätzlich haben die meisten der Befragten zum Befragungszeitpunkt schon einmal etwas über Reproduktionsmedizin gehört, gesehen oder gelesen. Erwartungsgemäß ist dies bei Frauen und wahrscheinlich bei den Probanden, bei welchen die Realisierung des Kinderwunsches besonders präsent ist, noch häufiger der Fall (vgl. Abbildung 3). 
$A b b$. 2: Wissen zur Fruchtbarkeit: Ab welchem Alter nimmt die Fruchtbarkeit der Frau langsam ab?

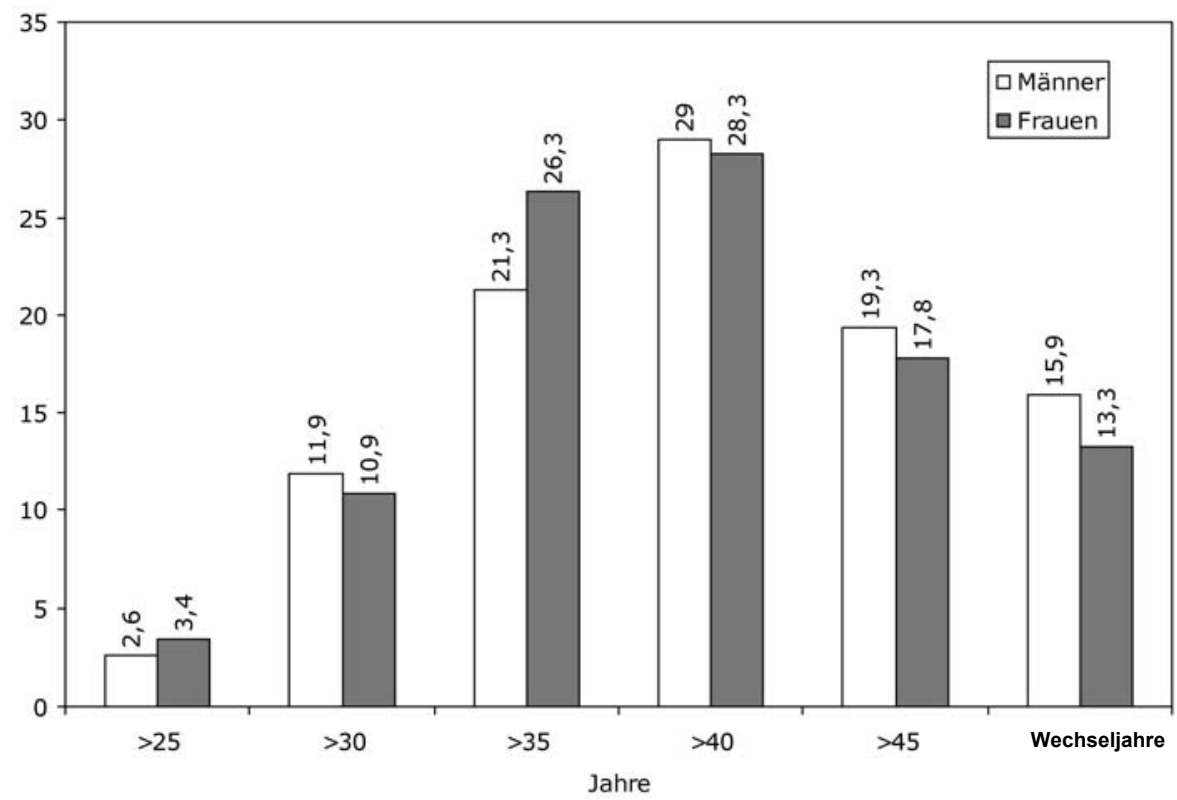

Quelle: Stöbel-Richter \& Brähler, 2005

$A b b$. 3: Kenntnis der Fortpflanzungsmedizin in der Allgemeinbevölkerung

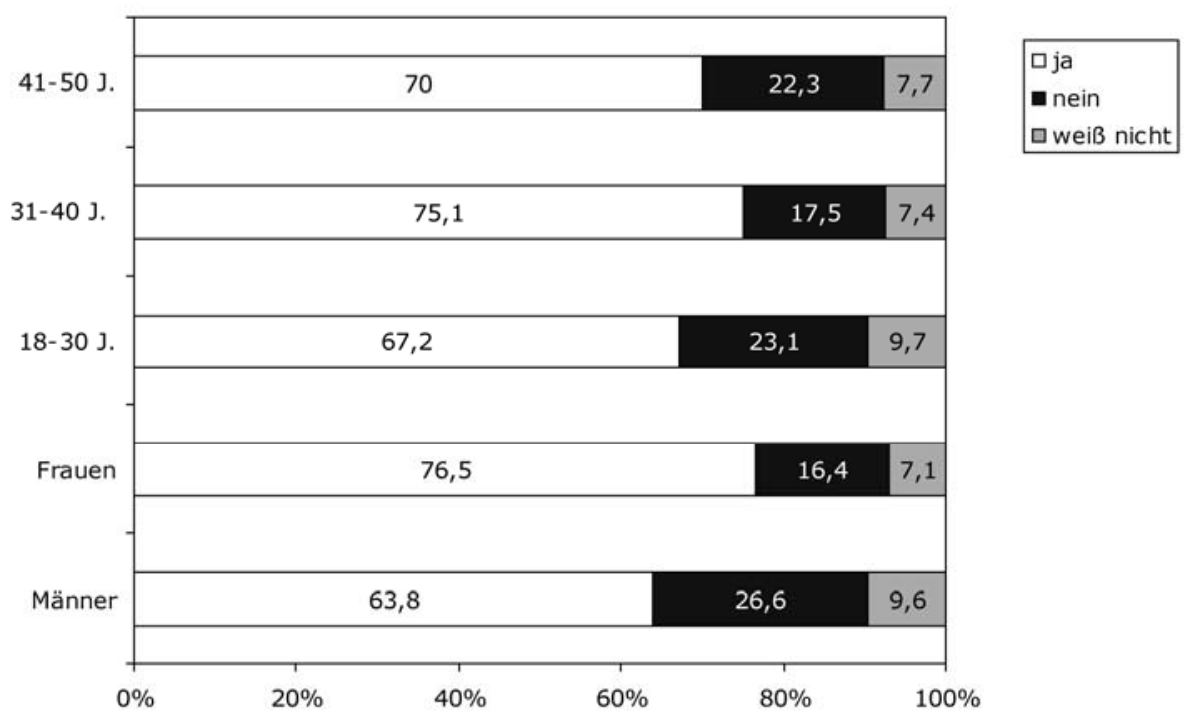

Quelle: Stöbel-Richter \& Brähler, 2005 
Die Befragten sollten einschätzen, wie hoch die Zahl der ungewollt kinderlosen Paare in Deutschland ist. Grundsätzlich wird diese als zu hoch eingeschätzt, ebenso wie die eingeschätzten Erfolgsraten einer reproduktionsmedizinischen Behandlung (vgl. Abbildung 4). Die meisten Befragten aus der repräsentativen Bevölkerungsstichprobe gehen von einer deutlich überschätzten Erfolgrate aus. Aber auch von den Paaren, die sich mit unerfülltem Kinderwunsch in eine Behandlung begeben, geben vor Behandlungsbeginn 50\% eine völlig überschätzte Erfolgsrate an (Daten aus dem Berliner Vergleichsprojekt, $\mathrm{N}=530$ Paare; Borkenhagen \& Kentenich, 2006).

Abb. 4: Erfolgsquote bei künstlicher Befruchtung

(,Wie hoch schätzen Sie die Erfolgsquote, also die Wahrscheinlichkeit ein, dass eine Frau bei einem Behandlungszyklus, bei dem sie künstlich befruchtet wurde, ein Kind zur Welt bringt?")
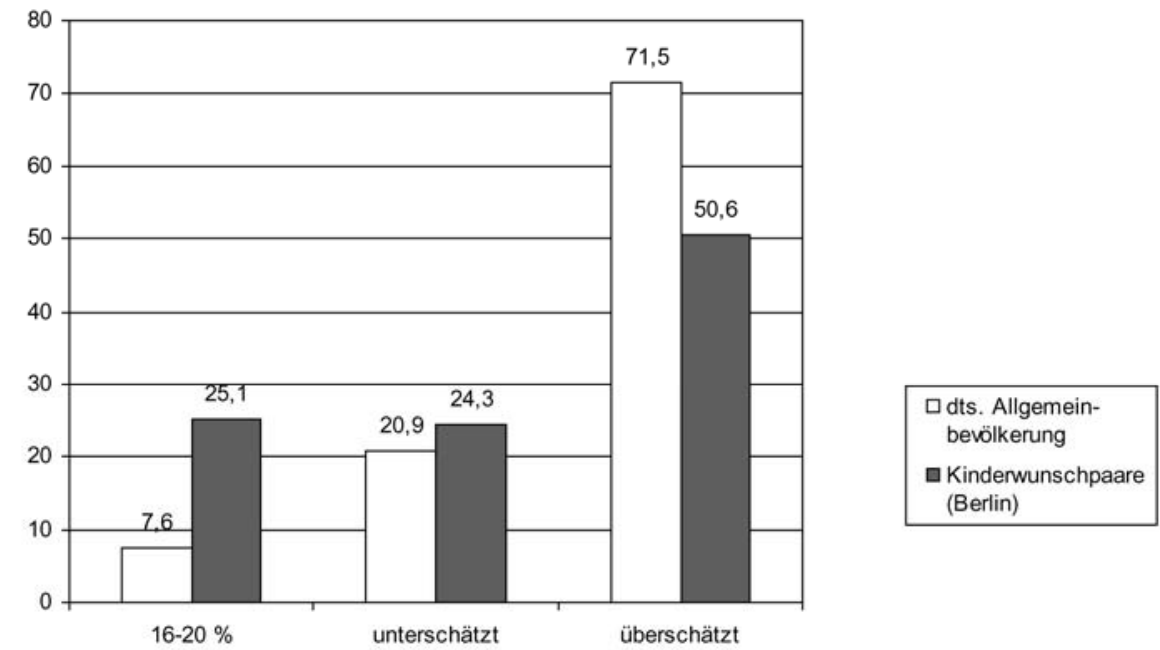

Quelle: zusammengefasste Darstellung. Brähler et al., 2005

Die reproduktionsmedizinischen Möglichkeiten werden deutlich überschätzt. Daher ist es auch nicht verwunderlich, dass 35,9\% der Frauen und 26,1\% der Männer angeben, dass sie im Falle einer ungewollten Kinderlosigkeit alle medizinisch möglichen Verfahren nutzen würden (vgl. Abbildung 5).

$1,3 \%$ der Befragten haben bereits reproduktionsmedizinische Maßnahmen in Anspruch genommen (Stöbel-Richter \& Brähler, 2005). Gegenwärtig werden in Deutschland ca. 3\% aller Kinder mit Hilfe reproduktionsmedizinischer Maßnahmen gezeugt (Strauß, Brähler \& Kentenich, 2004). Diese Zahlen können allerdings aufgrund geänderter Regelungen zur Kostenübernahme der Behandlung durch die Krankenkassen demnächst rückläufig sein. Ohne das hinter einem unerfüllten Kinderwunsch stehende Leid herunterspielen zu wollen, sollten doch aber in der Praxis die mit reproduktionsmedizinischen Maßnahmen anfallenden Kosten kritisch diskutiert werden und eine Behandlung ,um jeden Preis“ hinterfragt werden. 
Abb. 5: Reaktion auf eigene Kinderlosigkeit

(„Einmal angenommen, Sie selbst oder Ihr(e) Partner(in) könnten auf ,natürlichem“ Wege keine Kinder bekommen, haben aber einen Kinderwunsch. Was würden Sie tun?", Angaben in Prozent.)

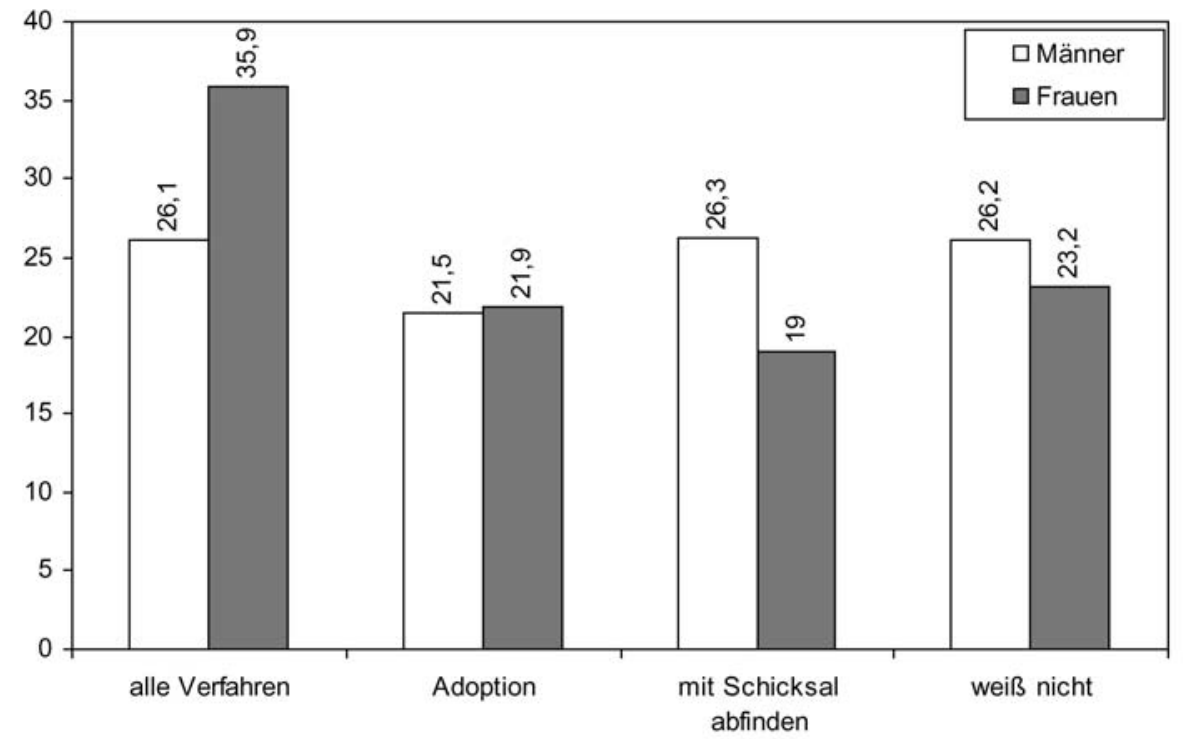

Quelle: Stöbel-Richter \& Brähler, 2005

\section{Individuelle Konsequenzen der Diagnose „Fertilitätsstörung“}

Wie wirkt sich die ungewollte Kinderlosigkeit im konkreten Einzelfall aus?

Viele Paare bringt die Diagnose einer Fertilitätsstörung an die Grenzen ihrer seelischen Belastbarkeit (Freeman, Boxer \& Rickels, 1985; Burns \& Covington, 1999; Carl; 2002). Gerade von Frauen kann die Tatsache, dass das „Natürlichste von der Welt nicht funktioniert“" (Spiewak, 2002, 26) als besonders kränkend empfunden werden. Studien aus den USA haben ergeben, dass Infertilität zu den stressvollsten Lebenssituationen gehören kann - vergleichbar mit dem Verlust eines Partners oder dem Tod eines Kindes. Die emotionalen Reaktionen auf die Diagnose „Fertilitätsstörung" reichen von Schock und Erstaunen bis hin zu Depression und Trauer (Spiewak, 2002). „Der unerfüllte Kinderwunsch als nicht-normatives Lebensereignis vermittelt Betroffenen den Eindruck, sozial in eine Außenseiterposition geraten zu sein" (Beyer, 2004).

In der sehr umfangreichen Arbeit von Beyer (2004) zur ungewollten Kinderlosigkeit, sowie deren Bewältigung werden sowohl kurz- als auch langfristige Folgen des unerfüllten Kinderwunsches unterschieden. Dabei zählen zu den kurzfristigen Folgen: eine starke emotionale Belastung, erhöhte Depressionswerte, Beeinträchti- 
gungen im Selbstwertgefühl, eine erhöhte Somatisierungsneigung und state-Ängstlichkeit sowie sozialer Rückzug und Stigmatisierungserfahrungen. Bei der Auswertung von längerfristigen Folgen konnten zum Teil keine Unterschiede zu Personen mit Kindern und keine Beeinträchtigungen durch Einsamkeit trotz weniger umfassender sozialer Netzwerke festgestellt werden. Dennoch sind ungewollt kinderlose Frauen im Alter belasteter als ungewollt kinderlose Männer und es kommt bei beiden Partnern zu einer schlechteren Versorgung im Alter und bei Krankheit.

Grundsätzlich stellt der unerfüllte Kinderwunsch vor allem für Frauen eine starke emotionale Belastung dar. Oft konnten bei kinderlosen Frauen höhere Depressivitätswerte nachgewiesen werden, die mit zunehmender Behandlungsdauer weiter ansteigen (Beyer, 2004).

Aber ungewollte Kinderlosigkeit ist in den meisten Fällen kein punktuelles, an einen bestimmten Lebensabschnitt gebundenes Phänomen, sondern Ergebnis einer allmählichen Entwicklung. Der hierbei entstehende subjektive Leidensdruck und die damit verbundene Notwendigkeit, ein leibliches Kind zu bekommen, sind nicht zu unterschätzen und können schließlich auch die Fortpflanzungsmediziner unter starken Handlungsdruck setzen. So entsteht eine wechselseitige Beeinflussung, in welcher aber die individuell Betroffenen die weit reichenden Konsequenzen nicht abzuschätzen vermögen. Von vielen Paaren wird der Reproduktionsmediziner - bewusst oder unbewusst - in die Rolle des verbündeten, überkompetenten und potenten Dritten gedrängt, dem auch die Entscheidung über einen weiteren Behandlungszyklus angetragen wird.

Vielfach werden nicht nur die Erfolgsraten, sondern auch die physischen und psychischen Belastungen einer reproduktionsmedizinischen Behandlung deutlich unterschätzt (van Balen, Naaktgeboren \& Trimbos-Kemper, 1996; Onnen-Isemann, 2000; Waldschmidt, 2002). Wie bereits oben gezeigt wurde, beendet die Mehrzahl aller Paare die reproduktionsmedizinische Behandlung erfolglos, vor allem dann, wenn die Frauen bereits älter als 40 Jahre sind (Brähler \& Stöbel-Richter, 2002). Liegen die klinischen Schwangerschaftsraten bzw. Raten des Embryotransfers bei mit IVF oder ICSI behandelten Frauen zwischen 31-35 Jahren noch bei 35\%, so sinken sie bei Frauen über 40 Jahre auf 10\% (DIR 2005). Dabei meinen diese Raten lediglich die klinischen Schwangerschaften, nicht jedoch die tatsächlichen Geburten, hierfür dürften die Zahlen noch niedriger sein, da mit höherem Alter auch die Abort- und Missbildungswahrscheinlichkeit erhöht ist. Auf die Themen Interruptio nach Pränataldiagnostik (nach fortpflanzungsmedizinischer Behandlung) oder Fruchtsackreduktion bei Mehrlingsschwangerschaften soll, trotz deren ethischer Brisanz, an dieser Stelle nicht eingegangen werden (vgl. hierzu Borkenhagen, Stöbel-Richter, Brähler \& Kentenich, 2004).

Sterilitätsaspekte spielen im Lebensentwurf nur äußerst selten eine Rolle, wie z.B. bei onkologischen Erkrankungen. „Normalerweise dreht sich die Kinderfrage um das $\mathrm{Ob}$ und Warum oder das Wann und mit Wem. Darüber was wäre, wenn es nicht klappt, denkt niemand nach.... Die trügerische Gewissheit, jederzeit schwanger werden zu können, lässt die Familiengründung planbar erscheinen. ... Je schöner der Plan, je enger das Zeitfenster zum Kinderkriegen, desto größer der Druck, wenn es nicht klappt“" (Spiewak, 2002, 29). Plötzlich sehen sich die Betroffenen gezwungen, zweigleisig zu leben - mit einem Plan für den Fall, dass alles wie bisher bleibt und ein Kind weiterhin auf sich warten lässt und mit einem zweiten Plan für das Eintre- 
ten der erhofften Schwangerschaft. Dennoch wird die Auseinandersetzung mit der Frage nach dem weiteren Leben, wenn der Kinderwunsch dauerhaft unerfüllt bleibt, erst einmal aufgeschoben. Gerade für beruflich erfolgreiche Paare, Mitte 30, die plötzlich die Erfahrung machen müssen, dass etwas in ihrem Leben nicht planbar ist und nicht auf Anhieb klappt, ist die Kränkung enorm. Der plötzliche Kontrollverlust über den eigenen Körper und somit über die vorgesehene Familienplanung stellt oftmals die erste schwere Lebenskrise dar.

Nichts desto trotz stellen sich „Kinderwunschpaare“ zu Behandlungsbeginn zufriedener und glücklicher dar, als Vergleichs- bzw. Kontrollgruppen (Felder et al., 2000; Goldschmidt, 1999; Beyer, 2004). Dies kann einerseits mit einem tatsächlichen stärkeren Zusammenhalt ob des gemeinsamen Leidensweges zusammenhängen. Anderseits muss aber auch, nicht zuletzt aufgrund zahlreicher eigener Interviewerfahrungen, geschlussfolgert werden, dass sich die Paare sehr sozial erwünscht darstellen und bei vielen die Angst vor einer weiteren Stigmatisierung eine kritische Reflektion der Paarbeziehung ausschließt. Hierzu schreiben Wischmann et al. (2002, 128): „Although they are often very distressed, at first many couples understandably shy away from speaking about their problems and conflicts. Many also fear that psychotherapists will find that there is an unconscious block, which could put the 'Blame' for the infertility on one ore both partners."

\section{Individuelle Aspekte der reproduktionsmedizinischen Behandlung}

Gegenwärtig trägt die Reproduktionsmedizin mit ca. $3 \%$ zu den Geburtenzahlen bei (Wischmann et al., 2004; Brähler et al., 2001a). Allerdings ist hier zu bemerken, dass es keine gesicherten Erkenntnisse darüber gibt, inwiefern es bei Nichtbehandlung oder durch andere Behandlungsmaßnahmen, z.B. eine Paartherapie, nicht auch zu ähnlichen Erfolgen gekommen wäre (Verres, Wischmann \& Gerhard, 2001).

Dennoch weckt jede neue Technik neue Hoffnungen und stellt damit die Betroffenen vor neue Entscheidungssituationen. Unter dem Motto „nichts unversucht gelassen zu haben" werden alle nur möglichen Techniken in Anspruch genommen. Auch wenn durch neue gesetzliche Regelungen hinsichtlich der Kostenübernahme die finanzielle Belastung hoch ist, können sich viele Paare nur schwer von ihren Hoffnungen und damit letztlich von ihrem Kinderwunsch verabschieden und eine erfolglose Behandlung akzeptieren und beenden.

Viele Frauen durchlaufen über Jahre hinweg reproduktionsmedizinische Behandlungen, die sie als körperlich und psychisch belastend empfinden. In körperlicher Hinsicht müssen sie sich mit Nebenwirkungen wie Kopfschmerzen, Schwindel, Übelkeit, Gewichtszunahme oder dem Risiko der Überstimulation auseinandersetzen. Bezogen auf die psychischen Belastungen ist vor allem der sich mit jeder Behandlung erneut wiederholende Kreislauf von Behandlung, Hoffen, Warten und Enttäuschung, wenn die Menstruation wieder einsetzt, zu verarbeiten. Die Frauen befinden sich oft in einem Spannungsfeld von Hoffen und ihrem Erleben, dass ihr Kinderwunsch unerfüllt bleibt (Sorg \& Fränznick, 2002; de Jong, 2002). 
Grundsätzlich ist die gesamte Behandlung eine Zeit der Geduld und des Wartens: warten, dass die Follikel reifen, warten, dass Ei- und Samenzellen sich vereinigen, warten auf das Ergebnis des Schwangerschaftstests und schließlich, wenn es nicht geklappt hat, warten auf den nächsten Versuch. Die Gefühle fahren während eines Behandlungszyklus Achterbahn: zum Beginn überwiegen Hoffnung und Optimismus, dann schleichen sich langsam Angst vor Misserfolg und wenn dieser bestätigt wird, Trauer und Verzweiflung ein. Als am schlimmsten wird von den meisten Frauen das Warten auf die Nachricht, ob die Behandlung erfolgreich war, beschrieben (vgl. Stammer, Verres \& Wischmann, 2004; Meyer \& Felder, 1998).

Obwohl bereits seit Jahren die psychologische Beratung und Aufklärungspflicht vor Behandlungsbeginn verbindlich per Gesetz geregelt wurden (5. Sozialgesetzbuch, \$27a, sowie Richtlinien der Bundesärztekammer (BÄK), vgl. hierzu auch Kentenich, 2001), zeigen sich in der Praxis erhebliche Diskrepanzen zwischen den verschiedenen Zentren. Inzwischen finden sich immer mehr Zentren, in denen ein Psychologe/ eine Psychologin zum Behandlungsteam gehören und in welchen auch eine psychosomatische Behandlungsweise favorisiert wird, dennoch gibt es auch nach wie vor Zentren, in denen die psychologische Beratung eher eine „Feuerlöscherfunktion“ in Notfällen hat und in denen eine strikt somatische, auf die punktuelle Diagnostik ausgerichtete Behandlung im Vordergrund steht (Kentenich et al., 2004). In letzteren werden dann auch Frauen unabhängig von ihrer psychischen und körperlichen Verfassung behandelt. „Fertilitätseinschränkungen werden in erster Linie als technische Probleme eingestuft, und die medizinischen Verfahren beziehen sich auf deren Lösung mithilfe bestimmter Techniken... Psychologische, soziale oder ökonomische Aspekte in der Verursachung oder als Folge der Kinderlosigkeit oder der Behandlung werden meist nicht oder nur am Rand berücksichtigt“" (Könnecke, 2000, 166).

Auch wenn jene Frauen, welche die Behandlung erfolglos abschließen oder diejenigen, die einen Abort erleiden, den größten Unterstützungsbedarf haben, ist eine psychologische Beratung während und nach der Behandlung für alle Patientinnen/ Paare nach wie vor einzufordern. Für diese so wichtige und doch auch sehr schmerzliche Zeit der Trauer fühlen sich die in den Zentren arbeitenden Reproduktionsmediziner nicht mehr zuständig. Somit steht ein enorm hoher medikamentöser, ärztlicher und finanzieller Aufwand, der betrieben wird, um eine Frau schwanger werden zu lassen, im krassen Missverhältnis zum Fehlen jeglicher Hilfe, wenn es nicht klappt.

Sowohl die Zeit der Behandlung, als auch die Zeit danach, stellen eine harte Belastungsprobe für das Paar dar. Phasen, in denen Schuldgefühle und entsprechende Vorwürfe verbalisiert werden, können ebenso auftreten, wie die gegenläufige Tendenz, dass das Paar durch die Behandlung noch enger zusammengeschweißt wird.

Die Ergebnisse der Studien des BMBF-Verbundes „Fertilitätsstörungen“ haben gezeigt, dass unmittelbar nach einer erfolglosen Behandlung 30-40\% der Frauen phasenweise in eine Depression fallen bzw. unter Ängstlichkeit und psychosomatischen Beschwerden leiden. Dabei kann aber davon ausgegangen werden, dass diese Symptome infolge des unerfüllten Kinderwunsches, die Diagnose der Unfruchtbarkeit und die Behandlung auftreten und nicht als psychische Auffälligkeiten a priori vorhanden sind (vgl. hierzu die Veröffentlichungen von Brähler \& Goldschmidt; 1998; Strauß, 2002 und Strauß, Brähler \& Kentenich, 2004). 
In verschiedenen Studien konnte aber auch gezeigt werden, dass vor allem Frauen, die über vielfältige personale bzw. soziale Ressourcen verfügen, die Behandlungsbelastungen positiver bewältigen, so z.B. Frauen mit einer optimistischen Grundhaltung, mit einem positiven Sinnerleben im unerfüllten Kinderwunsch, Frauen in der Auseinandersetzung mit der ungewollten Kinderlosigkeit eine Chance für die Partnerschaft sehen, sowie generell die realistische Einschätzung der Behandlungschancen. Frauen mit eher vermeidendem coping bzw. Frauen, die sich für den Ausgang der Behandlung verantwortlich fühlen und bei Misserfolg mit Schuld- und Schamgefühlen reagieren, sind eher gefährdet, in eine depressive Grundstimmung zu verfallen (vgl. Beyer, 2004).

So wie die Entscheidung für ein Kind ein Prozess ist, so ist auch die Verarbeitung einer ungewollten Kinderlosigkeit ein Prozess, der sich über lange Jahre hinziehen kann und dessen positiver Verlauf nicht zuletzt auch davon abhängt, die Trauer wirklich gespürt und zugelassen und Abschied von diesem Lebensentwurf genommen zu haben. Dabei spielen die Ursachenzuschreibungen eine wesentliche Rolle. „Kinderlose Frauen, die ihre Kinderlosigkeit im späten Erwachsenenalter durch äußere Umstände, wie beispielsweise das Schicksal oder die mangelnden medizinischen Möglichkeiten erklärten, waren mit ihrem Leben zufriedener als Frauen, die sich selbst die Schuld gaben“ (Beyer, 2004, 30).

Auch im Bereich der Forschungen zu ungewollter Kinderlosigkeit sind zahlreiche Forschungsdefizite vorhanden; vor allem über die Gruppe derjenigen, die sich trotz ungewollter Kinderlosigkeit nicht für eine Behandlung entschließen, ist bis heute nichts bekannt. Vielmehr konzentrieren sich die meisten Erhebungen auf Betroffene, bei denen es in Folge der Behandlung zur Elternschaft gekommen ist. Somit sind also auch die Erkenntnisse über die langfristige Verarbeitung der ungewollten Kinderlosigkeit lückenhaft (Beyer, 2004). Schließlich beruhen viele Studien auf relativ kleinen Fallzahlen, so dass eine Verallgemeinerung nur sehr schwer möglich ist.

\section{Ethisch-moralische und gesellschaftliche Aspekte}

Nicht nur das Bild der Elternschaft und die Rolle der Frau in der Gesellschaft werden durch die Methoden der medizinisch unterstützten Fortpflanzung tangiert, sondern auch die Sexualität, die Partnerschaft, die Schwangerschaft, die Rolle des Kindes und die Rolle des Mannes (Ulrich et al., 2000; Laster, Siedentopf \& Kentenich, 2000). Die Entwicklung der medizinischen Technik kann zur programmierten Zeugung im Labor unter Einbeziehung von individuellen und sozial akzeptierten Wunschkriterien führen, was z.B. bereits jetzt in einigen Ländern am Einsatz der PID zur Geschlechtswahl sichtbar wird. Durch die Trennung von Zeugung und Sexualität, wie ja bei reproduktionsmedizinischen Verfahren praktiziert, entzieht sich die Zeugung dem genetischen Zufallsprinzip. Die durch die Einführung der Pille mögliche Sexualität ohne Zeugung wurde von vielen als Befreiung empfunden, ob dies bei der fortschreitenden Trennung der Zeugung von der Sexualität auch der Fall ist, darf bezweifelt werden.

Durch das Hinausschieben der Erstelternschaft, vor allem in Akademikerkreisen, werden Argumentationen möglich, die eine Planbarkeit der Elternschaft mit Hilfe 
der Reproduktionsmedizin propagieren. Der Erfinder der „Pille“, Carl Djerassi, sprach sich in einem Interview dafür aus, dass Frauen und Männer im Alter von 20 Jahren ihre Ei- bzw. Samenzellen einfrieren lassen sollten, um diese dann zu gegebener Zeit - nach beruflicher und finanzieller Etablierung - wieder auftauen und mittels reproduktionsmedizinischer Verfahren befruchten und in die Frau einspülen zu lassen (Schindele, 2000). Dabei wird allerdings impliziert, dass dann auch eine Schwangerschaft zustande kommt. Schaut man sich aber die Bilanzen des deutschen IVF-Registers an, so wird schnell klar, dass für eine Frau ab 40 Jahren die Chancen auf eine erfolgreiche Behandlung mit erfolgreicher Schwangerschaft minimal sind.

Die im deutschen IVF-Register angegebenen Zahlen für eine erfolgreiche Behandlung betragen nach IVF 20,5\%, nach ICSI 24,7\%, meinen aber den Embryotransfer. Die dokumentierte (unvollständige) Lebendgeburtenrate lag 2002 für IVF bei 14,5\%, für ICSI bei 16,5\% (DIR, 2003). Für das Jahr 2004 gingen die Zahlen, aufgrund der neuen Zuzahlungsregelungen der Krankenkassen noch weiter zurück: nur noch 11.364 IVF-Zyklen und 23.942 ICSI-Behandlungen wurden durchgeführt. Davon bekamen nach einer IVF-Behandlung $11 \%$, nach einer ICSI-Behandlung 12\% der Frauen ein Kind (DIR, 2005, vgl. Tabelle 3). Wenn man nicht die klinischen Schwangerschaften $100 \%$ setzt, so wie das im Deutschen IVF-Register (DIR) üblich ist, sondern die durchgeführten Behandlungszyklen, so wird das ganze Ausmaß und die ganze Widersprüchlichkeit der Verfahren deutlich: lediglich 25\% können überhaupt eine erfolgreiche Behandlung im Sinne eines Embryotransfers verzeichnen und lediglich $11 \%$ bekommen schließlich auch ein Kind, d.h. von zehn Frauen verläuft für eine Frau die Behandlung wirklich erfolgreich!

Tabelle 3: IVF und ICSI - Durchgeführte Behandlungszyklen und Erfolgsraten (DIR 2004)

\begin{tabular}{lrrrr}
\hline 2004 & \multicolumn{1}{c}{ IVF } & \multicolumn{1}{c}{ ICSI } \\
\hline & Anzahl & \multicolumn{1}{c}{ Anzahl } & $\%$ \\
\hline Durchgeführte Behandlungen & 11.848 & 100 & 25.339 & 100 \\
Klinische Schwangerschaften (Implantation) & 2.971 & 25,07 & 6.658 & 26,27 \\
Keine Angaben & 974 & 8,22 & 2.083 & 8,22 \\
Geburten & 1.314 & 11,09 & 3.131 & 12,35 \\
Aborte & 626 & 5,28 & 1.334 & 5,26 \\
Extrauteringravidität & 57 & 0,48 & 110 & 0,43 \\
Einlinge & 1.008 & $76,71^{*}$ & 2.473 & $78,98^{*}$ \\
Zwillinge & 288 & $21,91^{*}$ & 621 & $19,83^{*}$ \\
Drillinge & 18 & $1,36^{*}$ & 37 & $1,18^{*}$ \\
\hline
\end{tabular}

* diese Prozentangaben sind auf die Geburten bezogen errechnet

Der HFEA-Report 2002 (Human Fertilisation and Embryology Authority) weist für den Zeitraum 2000/2001 für ICSI und IVF eine Erfolgsrate von 24,9\% aus. Die geringeren Raten in Deutschland sind auch durch die - im Anbetracht der methodischen Fortschritte - restriktiven Vorgaben des „Embryonenschutzgesetzes“ von 1990 bedingt. In den durch das Bundesministerium für Bildung, Forschung und Technik (BMBF) geförderten Forschungsverbünden des Förderschwerpunktes „Fertilitätsstörungen" waren es lediglich $30 \%$ aller mit reproduktionsmedizinisch assistierten Maßnahmen behandelten Frauen, die ihre Behandlung erfolgreich abschlossen. 
Neben diesem enormen finanziellen, materiellen, personellen und seelisch-körperlichen Aufwand, welcher für den erfolgreichen Abschluss einer Behandlung betrieben wird, muss auch mehr und mehr die soziokulturelle und gesellschaftliche Problematik der Reproduktionsmedizin gesehen werden; der oben beschriebene Aufschub der Erstelternschaft bringt verschiedene Konsequenzen mit sich: einen stärkeren Druck für die Frauen/Paare, wenn es nicht gleich klappt, somit eine frühere Inanspruchnahme von und eine stärkere Nachfrage nach reproduktionsmedizinischen Maßnahmen und die Weitergabe des Drucks auf die Reproduktionsmediziner, welche einerseits häufig die realen Chancen einer erfolgreichen Behandlung nicht klar darlegen und die andererseits immer stärker nach Möglichkeiten suchen, die Erfolgsraten der Verfahren zu verbessern (z.B. durch Micro-Sort - s. Glossar - oder PID). So ist es denn auch nicht verwunderlich, dass eine durch IVF oder ICSI schwanger gewordene Frau grundsätzlich als Risikoschwangere eingestuft wird, was eine vermehrte pränatale Diagnostik (auch invasiv) und häufigere Geburten per Kaiserschnitt nach sich zieht.

\section{Allgemeine Bewertung der Reproduktionsmedizin in der Bevölkerung}

Wie werden verschiedene, auch im Text angesprochene Aspekte der Reproduktionsmedizin in der Allgemeinbevölkerung bewertet? Den im Jahr 2003 in der o.g. repräsentativen Bevölkerungsbefragung befragten Personen wurden mehrere Aussagen zu verschiedenen Aspekten der Reproduktionsmedizin vorgegeben:

1 Ungewollt kinderlose Paare sollten alle Techniken der Fortpflanzungsmedizin nutzen, um leibliche Kinder zu bekommen.

2 Unfruchtbare Paare nehmen Techniken der Fortpflanzungsmedizin in Anspruch, ohne die Risiken wirklich abschätzen zu können.

3 Auch ältere Frauen sollten unabhängig von der biologischen Altersgrenze ihren Kinderwunsch mit Hilfe der Fortpflanzungsmedizin verwirklichen können.

4 Die Anwendung neuer Techniken in der Fortpflanzungsmedizin verursacht schwerwiegende moralische Konflikte.

Die Antworten erfolgten auf einer fünfstufigen Skala von $1=$ stimme nicht zu bis 5 = stimme voll zu. In Abbildung 6 sind die Antworten ,stimme nicht zu“ und ,stim-

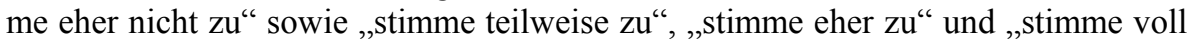

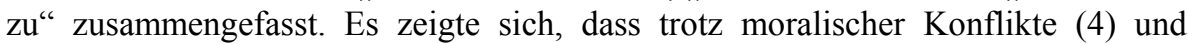
schwer abzuschätzender Risiken (2) ungewollt kinderlose Paare alle verfügbaren Techniken nutzen sollten (1), ältere Frauen nach dem Klimakterium diese Chancen jedoch nicht wahrnehmen sollten (3) (s. Abbildung 6). 
$A b b$. 6: Meinungen zu verschiedenen Aspekten der Reproduktionsmedizin

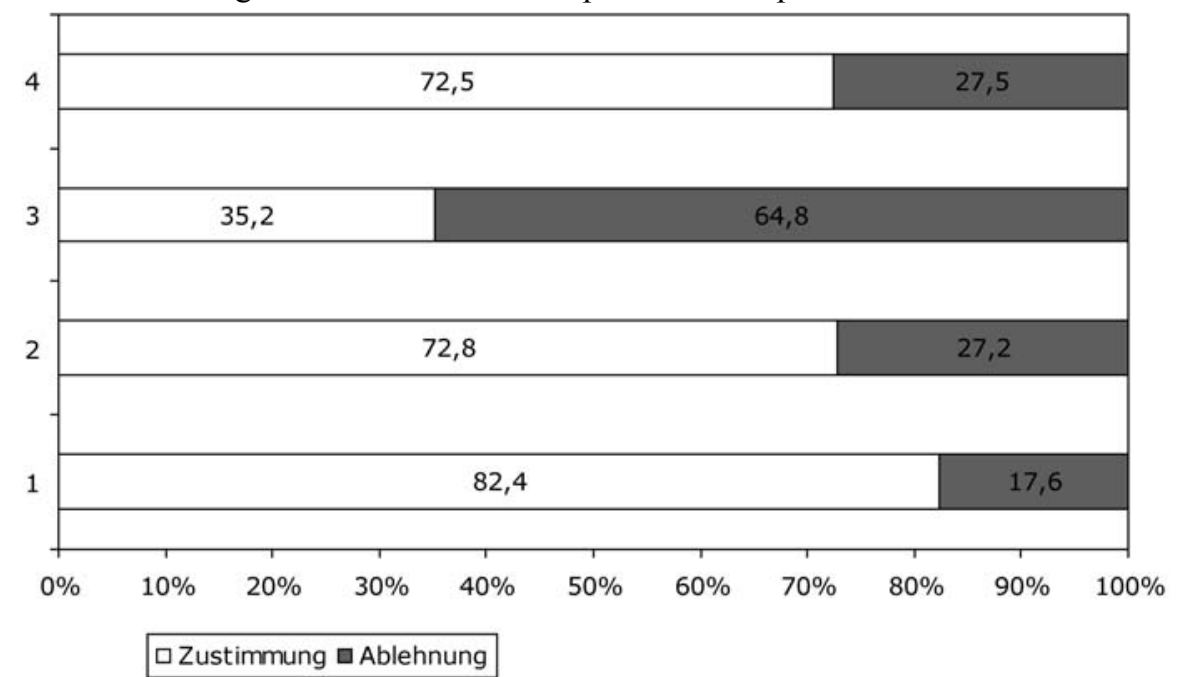

1 Ungewollt kinderlose Paare sollten alle Techniken der Fortpflanzungsmedizin nutzen, um leibliche Kinder zu bekommen.

2 Unfruchtbare Paare nehmen Techniken der Fortpflanzungsmedizin in Anspruch, ohne die Risiken wirklich abschätzen zu können.

3 Auch ältere Frauen sollten, unabhängig von ihrer biologischen Altersgrenze, ihren Kinderwunsch mit Hilfe der Fortpflanzungsmedizin verwirklichen können.

4 Die Anwendungen neuer Techniken in der Fortpflanzungsmedizin verursacht schwerwiegende moralische Konflikte.

Quelle: Stöbel-Richter \& Brähler, 2005

\section{Zusammenfassung}

Die Zahl ungewollt kinderloser Paare in Deutschland ist in der Vergangenheit mit $15-20 \%$ deutlich überschätzt worden. Der Prozentsatz dieser Paare liegt in Deutschland aktuell unter 3\%. Im Gegensatz dazu steigt die Zahl der gewollt kinderlosen Paare. Dabei korrespondiert der höhere Bildungsgrad der Frauen zum Teil mit einem höheren Ausmaß an Kinderlosigkeit. Diese Kinderlosigkeit ist maßgeblich durch den Aufschub der Realisierung des Kinderwunsches bedingt, das durchschnittliche Gebäralter von Frauen zwischen 15 und 49 Jahren lag in Deutschland im Jahr 2001 bei 28,8 Jahren, die Tendenz steigt weiterhin (Eurostat, 2005).

In Deutschland wünschen sich die meisten Menschen nach wie vor zwei Kinder. Dass dies nicht verwirklicht wird, kann auch auf eine zunehmende Elternfeindlichkeit unserer Gesellschaft zurückgeführt werden. So ergab eine neue Repräsentativbefragung der 16- bis 49-jährigen Bevölkerung in Frankreich und Deutschland des Instituts für Demoskopie Allensbach vom April 2007 mit dem Titel „Land ohne Kinder? Geburtenrate - ein deutsch-französischer Vergleich“ dass $80 \%$ der franzö- 
sischen Bevölkerung zutiefst überzeugt sind, in einem kinderfreundlichen Land zu leben. Im Gegensatz dazu sind dies in Deutschland laut der Umfrage nur 25\%. Eine Mehrheit der deutschen Bevölkerung hält Deutschland sogar für wenig kinderfreundlich. Die Ergebnisse zeigen weiterhin, dass auch die persönlichen Kinderwünsche in Frankreich signifikant höher liegen als in Deutschland - sowohl bei den Kinderlosen als auch bei den Eltern. Die Ergebnisse unterstreichen die Ergebnisse der Population Policy Acceptance Study (PPAS) 2003 und des Generations and Gender Survey (GGS) 2005 (Dorbritz, Lengerer \& Ruckdeschel, 2005; Höhn, Ette \& Ruckdeschel, 2006; Ruckdeschel, 2007; Gückel, 2007).

Hier muss in Deutschland also kein Wertewandel bei den jungen Menschen stattfinden, sondern ein Wandel bei der Setzung der gesellschaftlichen Rahmenbedingungen. $\mathrm{Ob}$ das in diesem Jahr eingeführte neue Elterngeld hierbei ein erster Schritt sein kann, wird sich noch zeigen müssen. Dass derartige Maßnahmen die Geburtenraten steigern können, ebenso wie die Bereitstellung von Betreuungsplätzen und damit die Erleichterung der Vereinbarkeit von Beruf und Familie oder auch die steuerlichen Begünstigungen für unverheiratete Paare mit Kindern, zeigen die Geburtenziffern in Ländern wie Frankreich, Norwegen und Schweden (Hoem, Neyer \& Andersson, 2006).

Es wird jungen Leuten fälschlich suggeriert, dass sie ihren Lebensplan beliebig gestalten und später noch ein Kind bekommen können. Die Polemik der Medien, dass mit Hilfe der assistierten Reproduktionsmedizin problemlos der hinausgeschobene Kinderwunsch nahezu unabhängig vom Alter der Frau realisiert werden kann, erweist sich in den meisten Fällen als trügerisch.

Reproduktionsmedizinische Verfahren sind in der Bevölkerung relativ bekannt, allerdings sehr ungenau. Die unterstreicht nur den Beratungsbedarf bei Paaren, die eine Behandlung wünschen. Auch wenn ungewollte Kinderlosigkeit als belastendes Lebensereignis unbedingt akzeptiert werden muss, so sollte das erste Ziel einer jeglichen Sterilitätstherapie immer sein, die spontanen Konzeptionsraten zu erhöhen (Kentenich et al., 2004).

Besonders schwerwiegend sind Aborte nach einer Kinderwunschbehandlung, hier besteht weiterhin Unterstützungsbedarf, welchem in der Praxis oft nicht gerecht wird. Die Inanspruchnahme einer psychologischen Beratung steigt mit der Kinderwunschdauer und der hohen Belastung durch den unerfüllten Kinderwunsch (Wischmann, 2003). Da Infertilität auch stets mit psychischen Belastungen verbunden ist, sollten die Bestrebungen in der Praxis vor allem dahin gehen, dass die psychosoziale Beratung ein integraler Bestandteil des Behandlungsangebotes ist. Dazu ist es wichtig, dass neben der Beratung entsprechend der psychosomatischen Grundversorgung durch den Arzt ein behandlungsunabhängiges Angebot getrennt von der ärztlichen Betreuung stattfindet. Dieses Angebot muss durch geschultes, d. h. über die körperlichen und psychischen Aspekte der Infertilität gut informiertes Personal, erfolgen. Neben der fachkundigen psychosomatischen Begleitung eines Paares kann diese Beratung auch dazu dienen, stark belastete Paare, die einer weiteren psychotherapeutischen Betreuung bedürfen, rechtzeitig zu identifizieren (Strauss, Brähler \& Kentenich, 2004).

Der Entscheidungsprozess für oder gegen ein Kind unterliegt nicht nur individuellen Werten und Rahmenbedingungen, sondern wird auch durch gesellschaftlich- 
strukturelle Aspekte determiniert. Auf der individuellen Ebene gilt es, sich mit vielfältigen Ambivalenzen auseinanderzusetzen, ein Prozess, welcher mit dem Eintritt einer Schwangerschaft durchaus nicht beendet sein muss. Unsere Daten zeigen, dass nicht jede Schwangerschaftsplanung erfolgreich realisiert wird bzw. werden kann, denn der Kinderwunsch allein macht noch nicht schwanger.

\section{Literatur}

Arbeitskreis Deutscher Marktforschungsinstitute (ADM) (1979). Musterstichprobenpläne. München: Moderne Industrie.

Bavister, B. D. (2002). Early history of in vitro fertilization. Reproduction, 124, pp. 181-196.

Beck, U. \& Beck-Gernsheim, E. (1990). Das ganz normale Chaos der Liebe. Frankfurt/M.: Suhrkamp.

Beck-Gernsheim, E. (2006). Die Kinderfrage heute. Über Frauenleben, Kinderwunsch und Geburtenrückgang. München: C. H. Beck.

Beutel, M. (2002). Der frühe Verlust eines Kindes. Bewältigung und Hilfe bei Fehl-, Todgeburt und Fehlbildung. Göttingen: Hogrefe.

Beyer, K. (2004). Ungewollte Kinderlosigkeit. Betroffene Frauen und ihre Bewältigung im mittleren Erwachsenenalter. Dissertation an der FSU Jena, Fakultät für Sozial- und Verhaltenswissenschaften.

BMFSFJ (Bundesministerium für Familie, Senioren, Frauen und Jugend) (2005). Deutschland: Kinderlos trotz Kinderwunsch? Monitor Familiendemographie 1.

Borkenhagen, A., Stöbel-Richter, Y., Brähler, E. \& Kentenich, H, (2004). Mehrlingsproblem bei Kinderwunschpaaren. Gynäkologische Endokrinologie, (2), pp. 163-168.

Borkenhagen, A. \& Kentenich, H. (2006). Einfluss der Möglichkeiten und der rechtlichen Situation auf die Einstellung und das Wissen zu kontroversen medizinischen und ethischen Fragen in der Reproduktionsmedizin - Wie entscheiden sich Personen in der konkreten Situation? Eine Studie an betroffenen Kinderwunschpaaren. Unveröffentlichter Abschlußbericht

Brähler, C. (1990). Familie, Kinderwunsch, Unfruchtbarkeit. Opladen: Westdeutscher Verlag.

Brähler, E. \& Goldschmidt, S. (1998). (Hrsg.). Psychosoziale Aspekte von Fruchtbarkeitsstörungen. Göttingen: Huber (Beiträge zur psychologischen Forschung).

Brähler, E., Stöbel-Richter, Y., Huinink, J. \& Glander, H.-J. (2001a). Zur Epidemiologie gewollter und ungewollter Kinderlosigkeit in Ost- und Westdeutschland. Reproduktionsmedizin, 17, S.157-162

Brähler, E., Stöbel-Richter, Y. \& Schumacher, J. (2001b). Für und Wider eines eigenen Kindes: Der Leipziger Fragebogen zu Kinderwunschmotiven (LKM). Diagnostica, 47, S. $96-$ 106.

Brähler, E. \& Stöbel-Richter, Y. (2002). Familienfeindlicher Zeitgeist? - Zum Wandel im Reproduktionsverhalten in Deutschland und im europäischen Vergleich. Reproduktionsmedizin, 18, S. 276-282.

Brähler, E. \& Stöbel-Richter, Y. (2003). Vermehren sich die Unfruchtbaren? - Eine Epidemiologie gewollter und ungewollter Kinderlosigkeit in Deutschland. In: M. Neises, S. Bartsch, H. Dohnke, H.-R. Falck, W. Kauffels, G. Schmidt-Ott, J. Schwerdtfeger \& H. Walter (Hrsg.), Psychosomatische Gynäkologie und Geburtshilfe. Beiträge der Jahrestagung 2002 der DGPFG. Gießen: Psychosozial-Verlag, S. 482-489.

Brähler, E., Stöbel-Richter, Y., Borkenhagen, A., Kentenich, H., Wisch, S. \& Strauß, B. (2005). Einstellungen und Wissen zu kontroversen medizinischen und ethischen Fragen in 
der Reproduktionsmedizin und der Präimplantationsdiagnostik. Vortrag auf dem Statuskolloquium des BMBF 2005 in Berlin.

Burkart, G. (1994). Die Entscheidung zur Elternschaft. Eine empirische Kritik von Individualisierungs- und Rational-Choice-Theorien. Stuttgart: Enke.

Burns, L.H., Covington, S.H. (1999) (Eds). Infertility counseling. A comprehensive handbook for clinicans. New York (Parthenon Publishing).

Carl, C. (2002). Leben ohne Kinder. Wenn Frauen keine Mütter sein wollen. Reinbek bei Hamburg: Rowohlt.

Cornelißen, W. (2004). Lebensentwürfe junger Frauen. Gynäkologe, 37, S. 585-590.

Crosignani, P. G. \& Rubin, B. (1996). Infertility revisited: The state of the art today and tomorrow. In: Guidelines. Prevalence, Diagnosis, Treatment and Management of Infertility - The ESHRE Capri Workshop. Experts on Human Reproduction, 4, pp. 5-7.

De Jong, T. M. (2002). Babys aus dem Labor. Segen oder Fluch? Weinheim (Beltz).

Dickmann, N. (2003). Demographischer Wandel - Geburtenraten im internationalen Vergleich. IW-Trends, 1, S. 1-25.

DIR (2003)/ (2005). Deutsches IVF-Register für das Jahr 2003. Bad Segeberg: Bundesgeschäftsstelle, Ärztekammer Schleswig Holstein.

DIW (2004). Kinderlose Männer in Deutschland - eine sozialstrukturelle Bestimmung auf Basis des Sozio-ökonomischen Panels (SOEP). Pressemitteilung des DIW Berlin vom 27.01.2004.

Dorbritz, J. (2005). Kinderlosigkeit in Deutschland und Europa - Daten, Trends und Einstellungen. Zeitschrift für Bevölkerungswissenschaften, 30, S. 359-408.

Dorbritz, J., Lengerer, A., Ruckdeschel, K. (2005). Einstellungen zu demographischen Trends und zu bevölkerungsrelevanten Politiken. Wiesbaden: Bundesinstitut für Bevölkerungswissenschaften.

Edwards, R. G., Bavister, B. D., Steptoe, P. C. (1969). Early stages of fertilisation in vitro of preovulatory human oocytes. Nature, 227, pp. 1307-1309.

ESHRE Capri Workshop Group (2001). Social determinants of human reproduction. Human Reproduction, 16, pp. 1518-1526.

Eurostat (2003). Erste Ergebnisse der Erhebung von Bevölkerungsdaten in Europa 2002.

Eurostat (2005). Europa in Zahlen. Eurostat Jahrbuch 2005. Luxemburg: Amt für Veröffentlichungen der Europäischen Gemeinschaften.

Felder, H., Pantke-Ehlers, E. \& Meyer, F. (2000). Subjektive Köperbeschwerden von Frauen und Männern während der In-vitro-Fertilisation. In: E. Brähler, H. Felder \& B. Strauss, (Hrsg.), Psychosoziale Aspekte der ungewollten Kinderlosigkeit. Göttingen: Hogrefe (Jahrbuch der Medizinischen Psychologie Band 17), S. 102-123.

Felder, H., Goldschmidt, S. \& Brähler, E. (2002). Prognostische Kriterien für das Eintreten von Schwangerschaften bei ungewollt kinderlosen Paaren. Reproduktionsmedizin 18(1), S. 15-24.

Freeman, E. W., Boxer, A. S., Rickels, K. (1985). Psychological evaluation and support in a program of in vitro fertilization and embryo transfer. Fertility and Sterility, 43, pp. 48-53.

Frick-Bruder, V. \& Schütt, E. (1992). Zur Psychologie des männlichen und weiblichen Kinderwunsches. Entwicklungsbedingungen narzißtischer, depressiver und kreativer Anteile. Psychotherapie, Psychosomatik, medizinische Psychologie, 42, S. 221-227.

Gloger-Tippelt, G. (1994). Psychologische Veränderungen beim Übergang zur Elternschaft. In: Frick-Bruder, V., Kentenich, H. \& Scheele, M. (Hrsg.). Psychosomatische Gynäkologie und Geburtshilfe. Beiträge zur Jahrestagung 1994. Gießen: Psychosozial-Verlag, S. 55-68.

Gloger-Tippelt, G., Gomille, B. \& Grimmig, R. (1993). Der Kinderwunsch aus psychologischer Sicht. Opladen: Leske + Budrich. 
Goldschmidt, S. (1999). Persönlichkeit, Paarbeziehung und Lebenszufriedenheit ungewollt kinderloser Paare bei In-vitro-Fertilisation. Dissertation an der Universität Leipzig.

Grünheid, E. (2004). Junge Frauen in Deutschland: Bei hoher Ausbildung kinderlos? In: Stöbel-Richter, Y. \& Brähler, E. (Hrsg.). Demographischer Wandel. Psychosozial 1/2004, S, 35-46.

Gückel, B. (2007). Keine Kinder erwünscht? - Die Ergebnisse einer Studie des Instituts für Demoskopie Allensbach weisen Deutschland im Vergleich zu Frankreich als kinderfeindliches Land aus. BiB-Mitteilungen 2/ 2007, 19-21.

Human Fertilisation and Embryology Authority (HFEA). http://www.hfea.gov.uk $<21.01 .2008>$

Henning, G. \& Henning, M. (1992). Soziale Faktoren und Motivationen zum Kinderwunsch und zum Schwangerschaftsabbruch. Sozialmedizinische Studie 1976-1990. In: U. Körner (Hrsg.), Ethik der menschlichen Fortpflanzung. Stuttgart: Ferdinand Enke Verlag, S. 77100.

Hill \& Kopp (2000). Fertilitätsentwicklung: Trends, Erklärungen und empirische Ergebnisse. In: U. Müller, B. Nauck \& A. Diekmann, (Hrsg.). Handbuch der Demographie. Berlin: Springer, S. 729-750.

Hoem, J. M., Neyer, G., Andersson, G. (2006). Education and childlessness - The relationship between educational field, educational level, and childlessness among Swedish born in 1955-59. Demographic Research 14(15), S, 331-338.

Höhn, C., Ette, A. \& Ruckdeschel, K. (2006). Kinderwünsche in Deutschland. Konsequenzen für eine nachhaltige Familienpolitik. Bundesinstitut für Bevölkerungsforschung/Robert Bosch Stiftung.

Huinink, J. (2002). Familienentwicklung in europäischen Ländern: Zur Erklärung von Polarisierungsphänomenen. In: J. Dorbritz, \& J. Otto (Hrsg.), Familienpolitik und Familienstrukturen. BiB: Wiesbaden: Bundesinstitut für Bevölkerungsforschung (Materialien zur Bevölkerungswissenschaft, Bd. 108), S. 47-60.

Ittner, E., Himmel, W. \& Kochen, M. M. (2000). Ungewollte Kinderlosigkeit in der Hausarztpraxis: Beratungs- und Betreuungsbedarf. In: E. Brähler, H. Felder \& B. Strauß (Hrsg.), Fruchtbarkeitsstörungen. Göttingen:Hogrefe (Jahrbuch der Medizinischen Psychologie 17), S. 229-244.

Kemkes-Grottenthaler, A. (2003). Postponing or rejecting parenthood? Results of a survey among female academic professionals. Journal of Biosocial Science, 35, pp. 213-226.

Kentenich, H. (2001). Reproduktionsmedizin in Deutschland. Reproduktionsmedizin, 17, S. 277-283.

Kentenich, H., Siedentopf, F., Borkenhagen, A., Heeger-Schmidmaier, J. \& Wessel, J. (2004). Bedürfnisse von Frauen mit Kinderwunsch. Gynäkologe, 37, S. 600-606.

Könnecke, R. (2000). Bewältigungsmuster ungewollt kinderloser Männer. Frankfurt (VAS).

Kühler, T. (1989). Zur Psychologie des männlichen Kinderwunsches. Ein kritischer Literaturüberblick. Weinheim: Deutscher Studienverlag.

Küppers-Chinnow, M. \& Karmaus, W. (1997). Prävalenz von verminderter Fruchtbarkeit und Inanspruchnahme ärztlicher Hilfe. Geburtshilfe und Frauenheilkunde, 57, 89-95.

Lanzieri, G. \& Corsini, V. (2006). Bevölkerung und soziale Bedingungen. Statistik kurz gefasst, 1. Luxemburg: Eurostat/Amt für Veröffentlichungen der Europäischen Gemeinschaften.

Laster, F., Siedentopf, F. \& Kentenich, H. (2000). Gibt es einen Einfluß der subjektiven Befindlichkeit von Patientinnen auf die Schwangerschaftsrate bei IVF/ ICSI? In: K. Weidner, V. Hellmann, D. Schuster, C. Dietrich \& M. Neises (Hrsg.), Psychosomatische Gynäkologie und Geburtshilfe. Beiträge der Jahrestagung 2000 der DGPGG und der OGPGG. Gießen: Psychosozial. S. 213-216. 
Malin, M., Hemminki, E., Räikkönen, O., Sihvo, S. \& Perälä, M.-L. (2001). What do women want? Women's experiences of infertility treatment. Social Science and Medicine 53, pp. 123-133.

Meyer, F. \& Felder, H. (1998): Das Erleben einer In-Vitro-Fertilisationsbehandlung unter Berücksichtigung der Paarbeziehung. In: E. Brähler \& S. Goldschmidt (Hrsg.), Psychosoziale Aspekte von Fruchtbarkeitsstörungen. Göttingen: Huber, S. 50-62.

Mittag, O. \& Jagenow, A. (1985). Motive zu Schwangerschaft, Geburt und Elternschaft. Ergebnisse einer empirischen Untersuchung an verhütungswilligen Frauen. In: Psychotherapie, Medizinische Psychologie, 34, S. 20-24.

Neyer, G. (2005). Die Ausbildungsrichtung ist entscheidend. Demografische Forschung Aus erster Hand, 2/3, 3.

Onnen-Isemann, C. (2000). Ungewollte Kinderlosigkeit und die Auswirkungen der Reproduktionsmedizin: Der Fall Deutschland. Forum Qualitative Sozialforschung, Online Journal 1, 1. http://www.qualitative-research.net/fqs-texte/1-00/1-00onnen-isemann-d.htm, <22,01.2008>

Ruckdeschel, K. (2005). Determinanten des Kinderwunsches in Deutschland. Mitteilungen des Bundesinstituts für Bevölkerungsforschung 3, S. 4.

Ruckdeschel, K. (2007). Nachdenken über den Kinderwunsch. BiB-Mitteilungen 1/2007, S. 13-16

Salakos, N., Roupa, Z., Sotiropoulou, P. \& Grigoriou, O. (2004). Family planning and psychosocial support for infertile couples. The European Journal of Contraception and Reproductive Health Care 9, pp. 47-51.

Saleth, S. (2005). Späte Mutterschaft - ein neuer Lebensentwurf? Statistisches Monatsheft Baden-Württemberg, 11, S. 14-15.

Scharein, M. \& Unger, R. (2005). Kinderlosigkeit bei Akademikerinnen? Die Aussagekraft empirischer Daten zur Kinderlosigkeit bei Akademikerinnen. BiB-Mitteilungen, 2, S. 6-13.

Schneewind, K. \& Vaskovics, L. A. (1992). Optionen der Lebensgestaltung junger Ehen und Kinderwunsch. Stuttgart: Kohlhammer.

Schneewind, K. \& Vaskovics, L. A. (1997). Optionen der Lebensgestaltung junger Ehen und Kinderwunsch. Verbundstudie - Endbericht. Stuttgart: Kohlhammer.

Schindele, E. (2000). Weibliche Lebensentwürfe und moderne Reproduktionstechnologien. Vortragsmanuskript.

Siedentopf, F., Laster, F. \& Kentenich, H. (2001). Gibt es einen Einfluß psychosozialer Faktoren auf die Schwangerschaftsrate während Sterilitätstherapie? Zentralblatt für Gynäkologie 123, S.73-75.

Sobotka, T. (2005). Kinder nur aufgeschoben oder ganz aufgehoben? Demografische Forschung-Aus erster Hand, 2/1, S. 3.

Sorg, B. \& Fränznick, M. (2002). Frauen in der Reproduktionsmedizin: Hoffnungen - Entscheidungszwänge - Behandlungsspiralen. In: E. Brähler, Y. Stöbel-Richter \& U. Hauffe (Hrsg.), Vom Stammbaum zur Stammzelle. Gießen: Psychosozial. S. 75-96.

Spiewak, M. (2002). Wie weit gehen wir für ein Kind? Frankfurt (Eichborn).

Stammer, H., Verres, R. \& Wischmann, T. (2004). Paarberatung und -therapie bei unerfülltem Kinderwunsch. Göttingen: Hogrefe.

Stöbel-Richter, Y. (2000) Kinderwunsch als Intention. Zur Relevanz persönlicher und gesellschaftlicher Kinderwunschmotive als Prädiktoren des aktuellen Kinderwunsches. Berlin: Verlag Colloquium Psychoanalyse.

Stöbel-Richter, Y. (2007). Verhütung und Planbarkeit von Schwangerschaft bei Paaren. Unveröffentlichtes Manuskript.

Stöbel-Richter, Y. (2007). Fertilität und Partnerschaft - Familienbildungsprozesse im Lebensverlauf. Ergebnisse der Sächsischen Längsschnittstudie (SLS) zu Partnerschaft, Familiengründung, Elternschaft sowie zur Bewertung familienpolitischer Aspekte. Habilitationsschrift eingereicht bei der Medizinischen Fakultät derUniversität Leipzig. 
Stöbel-Richter, Y. \& Brähler, E. (2005). Chancen und Verzicht - Grenzen der Fortpflanzungsmedizin bei ungewollter Kinderlosigkeit, Dortmund: Humanitas Verlag (Berliner Medizinethische Schriften Heft 54).

Stöbel-Richter, Y., Berth, H. \& Hinz, A. (2006). Kinderwunsch in der Paarinteraktion: Determinanten, Kommunikation und Zusammenhang mit Rollenerwartungen In: A. Hinz, \& O. Decker (Hrsg.), Gesundheit im gesellschaftlichen Wandel. Altersspezifik und Geschlechterrollen. Gießen: Psychosozial, S. 47-60.

Stöbel-Richter, Y. \& Brähler, E. (2006). Ausgewählte Fakten zum politischen Lamento über sinkende Kinderzahlen. Journal für Reproduktionsmedizin und Endokrinologie, 3, 307314.

Stöbel-Richter, Y., Förster, P., Brähler, E. und Berth, H. (2007). Vom Single zur Familie. In: H. Berth, P. Förster E. Brähler \& Y. Stöbel-Richter (Hrsg.), Einheitslust und Einheitsfrust. Gießen: Psychosozial, S. 143-176

Strauß, B. (2002) (Ed.). Involuntary childlessness. Göttingen: Hogrefe.

Strauß, B., Brähler, E. \& Kentenich, H. (2004) (Hrsg.). Fertilitätsstörungen - Psychosomatisch orientierte Diagnostik und Therapie. Leitlinie und Quellentext. Stuttgart: Schattauer.

Turchi, B. A. (1991). How economics, psychology, and sociology might produce a unified theory of fertility and labour force participation. In: J. Siegers, J. de Jong-Gierveld \& E. van Imhoff, (Eds), Female labour market behaviour and fertility. A rational choice approach. Berlin: Springer, pp. 237-245.

Ulrich, D., Gagel, D. E., Pastor, V.-S., \& Kentenich, H. (2000). Partnerschaft und Schwangerschaft nach durch IVF-erfülltem Kinderwunsch: eine ganz normale Sache? In: E. Brähler, H. Felder \& B. Strauß (Hrsg.), Fruchtbarkeitsstörungen, Göttingen: Hogrefe, S. 146-164.

Van Balen, F., Naaktgeboren, N. \& Trimbos-Kemper, T.C.M. (1996). In-vitro-fertilization: the experience of treatment, pregnancy and delivery. Human Reproduction, 11, pp. 95-98.

Verres, R., Wischmann, T. \& Gerhard, I. (2001). „Heidelberger Kinderwunsch-Sprechstunde" - Psychosomatische Beratung von Paaren mit unerfülltem Kinderwunsch: Teilprojekte „Fortbildung “ und „Beratung“. Unveröffentlichter Abschlußbericht.

Waldschmidt, A. (2002). Agonie oder Autonomie - die Verheißungen der Reproduktionsmedizin. In: ReproKult Frauen Forum Fortpflanzungsmedizin in Kooperation mit der Arbeitsstelle Pränataldiagnostik/Reproduktionsmedizin beim Bundesverband für Körperund Mehrfachbehinderte e.V., S. 1-66.

Wirth, H. \& Dümmler, K. (2005). The influence of qualification on women's childlessness between 1970 and 2001 in Western Germany. Zeitschrift für Bevölkerungswissenschaft, 30, S. 313-336.

Wischmann, T. (2003). Psychosoziale Aspekte bei Fertilitätsstörungen. Der Gynäkologe, 36, S. 125-136.

Wischmann, T., Stöbel-Richter, Y., Goldschmidt, S., Henning, K., Beyer, K., Stammer, H., Verres, R., Kentenich, H., Brähler, E. \& Strauß, B. (2004). Quellentext zur Leitlinie Fertilitätsstörungen - Psychosomatisch orientierte Diagnostik und Therapie - Diagnostik. In: B. Strauß, E. Brähler \& H. Kentenich (Hrsg). Fertilitätsstörungen - Psychosomatisch orientierte Diagnostik und Therapie. Leitlinie und Quellentext. Stuttgart: Schattauer, S. 23-43

Wischmann, T. Stammer, H. Gerhard, I. \& Verres, R. (2002). Couple counseling and therapy for the unfulfilled desire for a child - The two-step approach of the "Heidelberg Infertility Consultation Service". In: Strauß, B. (Hrsg.), Involuntary Childlessness. Göttingen: Hogrefe, pp.127-149. 
Anschriften der Autorinnen/Adresses of the authors:

Dr. phil. Yve Stöbel-Richter (Korrespondenzautorin/Corresponding author)

Dr. phil. Susanne Goldschmidt

Dr. phil. Ada Borkenhagen

Dipl-Psych. Ute Kraus

Abteilung Medizinische Psychologie und Medizinische Soziologie

UKL Leipzig

Frauenklinik

Philipp-Rosenthal-Straße 55

D-04103 Leipzig

E-mail: yve.stoebel-richter@medizin.uni-leipzig.de dr.borkenhagen@web.de ute.kraus@medizin.uni-leipzig.de

Dr. med. habil. Kerstin Weidner

Oberärztin der Klinik und Poliklinik für Psychotherapie und Psychosomatik Universitätsklinikum C. G. Carus an der Technischen Universität Dresden Augsburgerstraße 9, Haus 110

D-01307 Dresden

E-mail: kerstin.weidner@uniklinikum-dresden.de 


\section{Glossar}

Embryonenschutzgesetz: Seit 1.1.1991 in Kraft, regelt die Herstellung und Verwendung von menschlichen Embryonen bei o.g. Fertilisationsverfahren. Danach ist ihre Erzeugung zu anderen Zwecken als dem einer Schwangerschaft verboten. Pro behandeltem weiblichen Zyklus dürfen nicht mehr als 3 Embryonen hergestellt und übertragen werden, ihre „Produktion auf Vorrat" ist untersagt. Weiterhin verboten sind Gentransfers, Klonen, Chimären und Hybridbildung.

Embryotransfer (ET): Übertragung des (extrakorporal/ in-vitro gezeugten) Frühembryonen in die Gebärmutter der Frau.

extrakorporal: außerhalb des Körpers.

Fertilität: Fruchtbarkeit.

Intrazytoplasmatische Spermieninjektion (ICSI): Injektion einer einzelnen Samenzelle direkt in die Eizelle mit Hilfe von so genannten Mikromanipulatoren bei Fällen von extrem eingeschränkter männlicher Zeugungsfähigkeit.

In-vitro-Fertilisation (IVF): sog. Reagenzglasbefruchtung. Befruchtung erfolgt nicht in der weiblichen Tube, sondern $\rightarrow$ extrakorporal. Durch hormonelle Stimulationsbehandlungen werden mehrere Eizellen zur Ausreifung gebracht, die $\rightarrow$ laparoskopisch gezielt ausgewählt und mit einer hohen Anzahl vitaler Spermien zur Befruchtung gebracht werden. Die Embryonen werden später in Uterus oder Tube übertragen ( $\rightarrow$ Embryotransfer). Klassische Indikation: Fehlen oder Funktionsstörungen beider Tuben (tubare Sterilität).

Laparoskopie: Bauchspiegelung, operativer Eingriff zur Beurteilung der Eileiterdurchgängigkeit und zu Sterilisationsoperationen am inneren Genitale.

Micro-Sort: Trennung von X- und Y-Spermatozoen für die präkonzeptionelle Geschlechtswahl

Präimplantationsdiagnostik (PID) (auch PGD = engl. Preimplantation Genetic Diagnosis): Verfahren der genetischen Untersuchung des Embryos nach künstlicher Befruchtung und vor dem Einsetzen in die Gebärmutter der Frau (Implantation). Einzelne Zellen des Embryos im Sechs- und Achtzellstadium werden hierzu im Rahmen einer $\rightarrow$ IVF-Behandlung zur Diagnose schwerer Erbkrankheiten entnommen. In Deutschland, Österreich und der Schweiz nicht erlaubt.

Pränataldiagnostik: Gesamtheit aller Untersuchungen zur Erfassung vorwiegend genetisch bedingter Fehlbildungen und Stoffwechselerkrankungen vor der Geburt. Direkte Methoden beinhalten die Untersuchung des Feten bzw. der Fruchthöhle. Mütterliche Blutuntersuchungen geben indirekte Hinweise auf fetale Störungen.

Sterilität: Nichteintreten einer Schwangerschaft bei einem Paar, das über zwei Jahre (lt. WHO) ungeschützten Verkehr ausübt. Der Gynäkologe unterscheidet die primäre Sterilität, bei der noch keine Schwangerschaft eingetreten ist, von der sekundären, bei der nach einer vorangegangenen Schwangerschaft keine weiteren Konzeptionen eintreten. 